\title{
GEOMETRIC APPROACH TO STABLE HOMOTOPY GROUPS OF SPHERES. KERVAIRE INVARIANTS. II
}

\author{
P. M. Akhmet'ev
}

UDC 515.164

\begin{abstract}
We present an approach to the Kervaire-invariant-one problem. The notion of the geometric $(\mathbb{Z} / 2 \oplus \mathbb{Z} / 2)$-control of self-intersection of a skew-framed immersion and the notion of the $(\mathbb{Z} / 2 \oplus$ $\mathbb{Z} / 4)$-structure on the self-intersection manifold of a $\mathbf{D}_{4}$-framed immersion are introduced. It is shown that a skew-framed immersion $f: M^{\frac{3 n+q}{4}} \rightarrow \mathbb{R}^{n}, 0<q \ll n$ (in the $\left(\frac{3 n}{4}+\varepsilon\right)$-range), admits a geometric $(\mathbb{Z} / 2 \oplus \mathbb{Z} / 2)$-control if the characteristic class of the skew-framing of this immersion admits a retraction of order $q$, i.e., there exists a mapping $\kappa_{0}: M^{\frac{3 n+q}{4}} \rightarrow \mathbb{R P}^{\frac{3(n-q)}{4}}$ such that this composition $I \circ \kappa_{0}: M^{\frac{3 n+q}{4}} \rightarrow \mathbb{R} \mathrm{P}^{\frac{3(n-q)}{4}} \rightarrow \mathbb{R P}^{\infty}$ is the characteristic class of the skew-framing of $f$. Using the notion of $(\mathbb{Z} / 2 \oplus \mathbb{Z} / 2)$-control, we prove that for a sufficiently large $n, n=2^{l}-2$, an arbitrarily immersed $\mathbf{D}_{4}$-framed manifold admits in the regular cobordism class (modulo odd torsion) an immersion with a $(\mathbb{Z} / 2 \oplus \mathbb{Z} / 4)$-structure.
\end{abstract}

\section{Self-Intersections of Immersions and Kervaire Invariants}

The Kervaire-invariant-one problem is an open problem in algebraic topology (for details, see $[4,7]$ ). We consider an approach to a solution of this problem based on results of P. J. Eccles (see [8]). For a geometrical approach, see also $[5,6]$.

Let $f: M^{n-1} \rightarrow \mathbb{R}^{n}, n=2^{l}-2, l>1$, be a smooth (generic) immersion of codimension 1 . Let us denote by $g: N^{n-2} \uparrow \rightarrow \mathbb{R}^{n}$ the immersion of a self-intersection manifold.

Definition 1. The Kervaire invariant of $f$ is defined as

$$
\Theta(f)=\left\langle w_{2}^{\frac{n-2}{2}} ;\left[N^{n-2}\right]\right\rangle,
$$

where $w_{2}=w_{2}\left(N^{n-2}\right)$ is the normal Stiefel-Whitney class of $N^{n-2}$.

This is an invariant of the regular cobordism classes of immersions $f$. This means that the Kervaire invariant is a well-defined homomorphism

$$
\Theta: \operatorname{Imm}^{\mathrm{sf}}(n-1,1) \rightarrow \mathbb{Z} / 2 .
$$

The normal bundle $\nu(g)$ of the immersion $g: N^{n-2} \rightarrow \mathbb{R}^{n}$ is a 2-dimensional bundle over $N^{n-2}$ equipped with a $\mathbf{D}_{4}$-framing. The classifying mapping $\eta: N^{n-2} \rightarrow K\left(\mathbf{D}_{4}, 1\right)$ of this bundle is well defined. The $\mathbf{D}_{4}$-structure of the normal bundle or the $\mathbf{D}_{4}$-framing is the prescribed reduction of the structure group of the normal bundle of the immersion $g$ to the group $\mathbf{D}_{4}$ corresponding to the mapping $\eta$. The pair $(g, \eta)$ represents an element in the cobordism group $\operatorname{Imm}^{\mathbf{D}_{4}}(n-2,2)$. The homomorphism

$$
\delta: \operatorname{Imm}^{\mathrm{sf}}(n-1,1) \rightarrow \operatorname{Imm}^{\mathbf{D}_{4}}(n-2,2)
$$

is well defined.

Let us recall that the cobordism group $\operatorname{Imm}^{\text {sf }}(n-k, k)$ generalizes the group $\operatorname{Imm}^{\text {sf }}(n-1,1)$. This group is defined as the cobordism group of triples $(f, \Xi, \kappa)$, where $f: M^{n-k} \rightarrow \mathbb{R}^{n}$ is an immersion with the prescribed isomorphism $\Xi: \nu(g)=k \kappa$ called a skew-framing, $\nu(f)$ is the normal bundle of $f$, and $\kappa$ is the given line bundle over $M^{m-k}$ with the characteristic class $w_{1}(\kappa) \in H^{1}\left(M^{m-k} ; \mathbb{Z} / 2\right)$. The cobordism relation of triples is standard.

Translated from Fundamentalnaya i Prikladnaya Matematika, Vol. 13, No. 8, pp. 17-41, 2007. 
The generalization of the group $\operatorname{Imm}^{\mathbf{D}_{4}}(n-2,2)$ is as follows. Let us define the cobordism groups $\operatorname{Imm}^{\mathbf{D}_{4}}(n-2 k, 2 k)$. This group $\operatorname{Imm}^{\mathbf{D}_{4}}(n-2 k, 2 k)$ is represented by triples $(g, \Xi, \eta)$, where $g: N^{n-2 k} \rightarrow \mathbb{R}^{n}$ is an immersion and $\Xi$ is a dihedral $k$-framing, i.e., the prescribed isomorphism $\Xi: \nu_{g}=k \eta$, where $\eta$ is a 2-dimensional bundle over $N^{n-2 k}$. The characteristic mapping of the bundle $\eta$ is also denoted by $\eta: N^{n-2 k} \rightarrow K\left(\mathbf{D}_{4}, 1\right)$ (a similar convention will be used). The mapping $\eta$ is the characteristic mapping for the bundle $\nu_{g}$ since $\nu_{g}=k \eta$.

Obviously, the Kervaire homomorphism (1) is defined as the composition of homomorphism (2) with a homomorphism

$$
\Theta_{\mathbf{D}_{4}}: \operatorname{Imm}^{\mathbf{D}_{4}}(n-2,2) \rightarrow \mathbb{Z} / 2 .
$$

Homomorphism (3) is called the Kervaire invariant for a $\mathbf{D}_{4}$-framed immersed manifold.

The Kervaire homomorphisms are defined in a more general situation by a straightforward generalization of homomorphisms (1) and (3):

$$
\begin{gathered}
\Theta^{k}: \operatorname{Imm}^{\text {sf }}(n-k, k) \rightarrow \mathbb{Z} / 2, \\
\Theta_{\mathbf{D}_{4}}^{k}: \operatorname{Imm}^{\mathbf{D}_{4}}(n-2 k, 2 k) \rightarrow \mathbb{Z} / 2
\end{gathered}
$$

(for $k=1$, the new homomorphism coincides with homomorphism (3) defined above) and the diagram

is commutative.

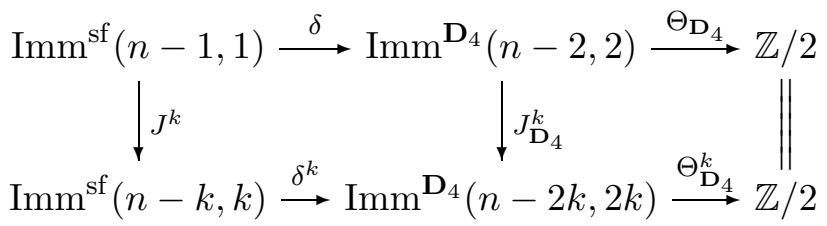

Let $(g, \Xi, \eta)$ be a $\mathbf{D}_{4}$-framed (generic) immersion in codimension $2 k$. Let $h: L^{n-4 k} \rightarrow \mathbb{R}^{n}$ be the immersion of the self-intersection (double-point) manifold of $g$. The normal bundle $\nu_{h}$ of the immersion $h$ is decomposed into the direct sum of $k$ isomorphic copies of the 4-dimensional bundle $\zeta$ with the structure group $\mathbb{Z} / 2 \prec \mathbf{D}_{4}$. This decomposition is given by the isomorphism $\Psi: \nu_{h}=k \zeta$. The bundle $\nu_{h}$ itself is classified by the mapping $\zeta: L^{n-4 k} \rightarrow K\left(\mathbb{Z} / 2\right.$ २ $\left.\mathbf{D}_{4}, 1\right)$.

All the triples $(h, \zeta, \Psi)$ described above (we do not assume that a triple is realized as the double-point manifold for a $\mathbf{D}_{4}$-framed immersion) up to the standard cobordism relation form the cobordism group $\operatorname{Imm}^{\mathbb{Z} / 2 \imath \mathbf{D}_{4}}(n-4 k, 4 k)$. The self-intersection of an arbitrary $\mathbf{D}_{4}$-framed immersion is a $\left(\mathbb{Z} / 2 \imath \mathbf{D}_{4}\right)$-framed immersed manifold and the cobordism class of this manifold well defines the natural homomorphism

$$
\delta_{\mathbf{D}_{4}}^{k}: \operatorname{Imm}^{\mathbf{D}_{4}}(n-2 k, 2 k) \rightarrow \operatorname{Imm}^{\mathbb{Z} / 2 \mathbf{D}_{4}}(n-4 k, 4 k) .
$$

The subgroup $\mathbf{D}_{4} \oplus \mathbf{D}_{4} \subset \mathbb{Z} / 2 \prec \mathbf{D}_{4}$ of index 2 induces the double cover $\bar{L}^{n-4 k} \rightarrow L^{n-4 k}$. This double cover corresponds with the canonical double cover over the double-point manifold.

Let $\bar{\zeta}: \bar{L}^{n-4 k} \rightarrow K\left(\mathbf{D}_{4}, 1\right)$ be the classifying mapping induced by the projection homomorphism $\mathbf{D}_{4} \oplus \mathbf{D}_{4} \rightarrow \mathbf{D}_{4}$ on the first factor. Let $\bar{\zeta} \rightarrow L^{n-4 k}$ be the 2-dimensional bundle defined as the pull-back of the universal 2-dimensional bundle with respect to the classifying mapping $\bar{\zeta}$.

Definition 2. The Kervaire invariant $\Theta_{\mathbb{Z} / 2 \imath \mathbf{D}_{4}}^{k}$ for a $\mathbb{Z} / 2 \prec \mathbf{D}_{4}$-framed immersion $(h, \Psi, \zeta)$ is defined by the following formula:

$$
\Theta_{\mathbb{Z} / 2\} \mathbf{D}_{4}}^{k}(h, \Psi, \zeta)=\left\langle w_{2}(\bar{\eta})^{\frac{n-4 k}{2}} ;\left[L^{n-4 k}\right]\right\rangle .
$$

This new invariant is a homomorphism $\Theta_{\mathbb{Z} / 2 l \mathbf{D}_{4}}^{k}: \operatorname{Imm}^{\mathbb{Z} / 2 \mathbf{D}_{4}}(n, n-4 k) \rightarrow \mathbb{Z} / 2$ included in the following commutative diagram:

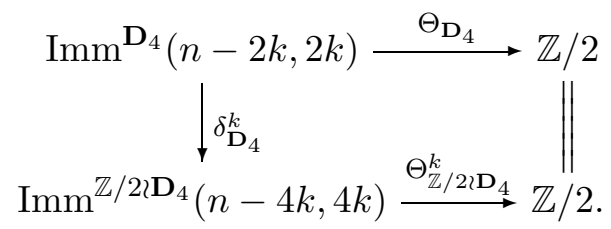


Let us formulate the main results of the paper. In Sec. 2 , the notion of $(\mathbb{Z} / 2 \oplus \mathbb{Z} / 2)$-control $\left(\mathbf{I}_{b}\right.$-control) on self-intersection of a skew-framed immersion is considered. Theorem 1 (for the proof see Sec. 3) shows that under a natural restriction of dimensions, the property of $\mathbf{I}_{b}$-control holds for an immersion in the regular cobordism class modulo odd torsion.

In Sec. 4 , we formulate the notion of $(\mathbb{Z} / 2 \oplus \mathbb{Z} / 4)$-structure of $(\mathbb{Z} / 2 \oplus \mathbb{Z} / 2)$-controlled immersion. In Sec. 5 (Theorem 2 ), we prove, under a natural restriction of dimension, that an arbitrary $(\mathbb{Z} / 2 \oplus \mathbb{Z} / 2)$-controlled immersion admits in the regular homotopy class an immersion with $(\mathbb{Z} / 2 \oplus \mathbb{Z} / 4)$-structure of self-intersection. For such an immersion, the Kervaire invariant is expressed in terms of $(\mathbb{Z} / 2 \oplus \mathbb{Z} / 4)$-characteristic numbers of the self-intersection manifold.

The author is grateful to Prof. M. Mahowald (2005) and Prof. R. Cohen (2007) for discussions and to Prof. A. A. Voronov for the invitation to the University of Minnesota in 2005.

This paper was begun in 1998 at the Postnikov Seminar and is dedicated to the memory of Prof. Yu. P. Solovyov.

\section{Geometric Control of Double Self-Intersection Manifolds of Skew-Framed Immersions}

In the following two sections, by $\operatorname{Imm}^{\text {sf }}(n-k, k), \operatorname{Imm}^{\mathbf{D}_{4}}(n-2 k, 2 k), \operatorname{Imm}^{\mathbb{Z} / 2 l \mathbf{D}_{4}}(n-4 k, 4 k)$, etc., we will denote not the cobordism groups itself, but the 2-components of this group. In the case where the first argument is strictly positive, all the groups are finite 2-groups.

Recall that the dihedral group $\mathbf{D}_{4}$ is given by the co-representation $\left\{a, b \mid a^{4}=b^{2}=e,[a, b]=a^{2}\right\}$. This group is the subgroup of the group $O(2)$, i.e., the transformation group of the standard plane with the base $\left\{f_{1}, f_{2}\right\}$. The element $a$ corresponds to the rotation of the plane by the angle $\frac{\pi}{2}$. The element $b$ corresponds to the reflection of the plane with respect to the axis given by the vector $f_{1}+f_{2}$.

Let $\mathbf{I}_{b}(\mathbb{Z} / 2 \oplus \mathbb{Z} / 2)=\mathbf{I}_{b} \subset \mathbf{D}_{4}$ be the subgroup generated by the elements $\left\{a^{2}, b\right\}$. The cohomology group $H^{1}\left(K\left(\mathbf{I}_{b}, 1\right) ; \mathbb{Z} / 2\right)$ is the elementary 2-group with two generators. The first (second) generator of this group detects the reflection of the second (first) coordinate axis, respectively. The generators of the cohomology group will be denoted by $\tau_{1}$ and $\tau_{2}$, respectively.

Definition 3. We say that a skew-framed immersion $(f, \Xi), f: M^{n-k} \rightarrow \mathbb{R}^{n}$, has a self-intersection of the type $\mathbf{I}_{b}$ if the double-point manifold $N^{n-2 k}$ of $f$ is a $\mathbf{D}_{4}$-framed manifold that admits a reduction of the structure group $\mathbf{D}_{4}$ of the normal bundle to the subgroup $\mathbf{I}_{b} \subset \mathbf{D}_{4}$.

Let us state the following conjecture.

Conjecture. For an arbitrary $q>0, q=2(\bmod 4)$, there exists a positive integer $l_{0}=l_{0}(q)$ such that for an arbitrary $n=2^{l}-2, l>l_{0}$, an arbitrary element $a \in \operatorname{Imm}^{\mathrm{sf}}\left(\frac{3 n+q}{4}, \frac{n-q}{4}\right)$ is stably regular cobordant to a stably skew-framed immersion with $\mathbf{I}_{b}$-type of self-intersection (for the definition of stable framing, see $[9]$; for that of stable skew-framing, see $[1,2])$.

Let us state and prove a weaker result toward the conjecture. We start with the following definition.

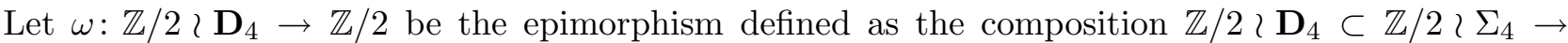
$\Sigma_{4} \rightarrow \mathbb{Z} / 2$, where $\Sigma_{4} \rightarrow \mathbb{Z} / 2$ is the parity of the transposition. Let $\omega^{!}: \operatorname{Imm}^{\mathbb{Z} / 2\left(\mathbf{D}_{4}\right.}(n-4 k, 4 k) \rightarrow$ $\operatorname{Imm}{ }^{\operatorname{Ker} \omega}(n-4 k, 4 k)$ be the transfer homomorphism induced by $\omega$.

Let $P$ be a polyhedron $\operatorname{dim}(P)<2 k-1, Q \subset P$ be a subpolyhedron, $\operatorname{dim}(Q)=\operatorname{dim}(P)-1$, and let $P \subset \mathbb{R}^{n}$ be an embedding. Let us denote by $U_{P}$ the regular neighborhood of $P \subset \mathbb{R}^{n}$ of radius $r_{P}$ and by $U_{Q}^{\prime}$ the regular neighborhood of $Q \subset \mathbb{R}^{n}$ of radius $r_{Q}, r_{Q}>r_{P}$. Let $U_{Q}=U_{P} \cap U_{Q}^{\prime}$.

The boundary $\partial U_{P}$ of the neighborhood $U_{P}$ is a codimension-one submanifold in $\mathbb{R}^{n}$. This manifold $\partial U_{P}$ is the union of two manifolds with boundaries $V_{Q} \cup_{\partial} V_{P}, V_{Q}=U_{Q} \cap \partial U_{P}$, and $V_{P}=\partial U_{P} \backslash U_{Q}$ along the common boundary $\partial V_{Q}=\partial V_{P}$.

Assume that two cohomological classes $\tau_{Q, 1} \in H^{1}(Q ; \mathbb{Z} / 2)$ and $\tau_{Q, 2} \in H^{1}(Q ; \mathbb{Z} / 2)$ are given. The projection $U_{Q} \rightarrow Q$ determines the cohomological classes $\tau_{U_{Q}, 1}, \tau_{U_{Q}, 2} \in H^{1}\left(U_{Q} ; \mathbb{Z} / 2\right)$ as the images of the classes $\tau_{Q, 1}$ and $\tau_{Q, 2}$, respectively. 
Let $\left(g, \Xi_{N}, \eta\right), \operatorname{dim}(N)=n-2 k$, be a $\mathbf{D}_{4}$-framed generic immersion, $n-4 k>0$, and $g\left(N^{n-2 k}\right) \cap \partial U_{P}$ be an immersed submanifold in $U_{Q} \subset \partial U_{P}$. Denote $g\left(N^{n-2 k}\right) \backslash\left(g\left(N^{n-2 k}\right) \cap U_{P}\right)$ by $N_{\text {int }}^{n-2 k}$, and the complement $N^{n-2 k} \backslash N_{\text {int }}^{n-2 k}$ by $N_{\text {ext }}^{n-2 k}$. The manifolds $N_{\text {ext }}^{n-2 k}$ and $N_{\text {int }}^{n-2 k}$ are submanifolds in $N^{n-2 k}$ of codimension 0 with a common boundary; this boundary is denoted by $N_{Q}^{n-2 k-1}$. The self-intersection manifold of $g$ is denoted by $L^{n-4 k}$. By dimension considerations $(n-4 k=q \ll n), L^{n-4 k}$ is a submanifold in $\mathbb{R}^{n}$ parameterized by an embedding $h$, equipped by the $\left(\mathbb{Z} / 2<\mathbf{D}_{4}\right)$-framing of the normal bundle denoted by $(\Psi, \zeta)$. The triple $(h, \Psi, \zeta)$ determines an element in the cobordism group $\operatorname{Imm}^{\mathbb{Z} / 2 l \mathbf{D}_{4}}(n-4 k, 4 k)$.

Definition 4. We say that the $\mathbf{D}_{4}$-framed immersion $g$ is an $\mathbf{I}_{b}$-controlled immersion if the following conditions hold:

(1) the structure group of the $\mathbf{D}_{4}$-framing $\Xi_{N}$ restricted over the submanifold (with boundary) $g\left(N_{\text {ext }}^{n-2 k}\right)$ is reduced to the subgroup $\mathbf{I}_{b} \subset \mathbf{D}_{4}$ and the cohomological classes $\tau_{U_{Q}, 1}, \tau_{U_{Q}, 2} \in$ $H^{1}\left(U_{Q} ; \mathbb{Z} / 2\right)$ are mapped to the generators $\tau_{1}, \tau_{2} \in H^{1}\left(N_{Q}^{n-2 k-1} ; \mathbb{Z} / 2\right)$ of the structure group of the $\mathbf{I}_{b}$-framing by the immersion $\left.g\right|_{N_{Q}^{n-2 k-1}}: N_{Q}^{n-2 k-1} \rightarrow \partial\left(U_{Q}\right) \subset U_{Q}$;

(2) the restriction of the immersion $g$ on the submanifold $N_{Q}^{n-2 k-1} \subset N^{n-2 k}$ is an embedding $\left.g\right|_{N_{Q}^{n-2 k-1}}: N_{Q}^{n-2 k-1} \subset \partial U_{Q}$ and the decomposition $L^{n-4 k}=L_{\text {int }}^{n-4 k} \cup L_{\text {ext }}^{n-4 k} \subset\left(U_{P} \cup \mathbb{R}^{n} \backslash U_{P}\right)$ of the self-intersection manifold of $g$ into two (probably, nonconnected) $\mathbb{Z} / 2 \imath \mathbf{D}_{4}$-framed components is well defined. The manifold $L_{\text {int }}^{n-4 k}$ is a submanifold in $U_{P}$ and the triple $\left(L_{\text {int }}^{n-4 k}, \Psi_{\text {int }}, \zeta_{\text {int }}\right)$ represents the element in $\operatorname{Imm}^{\operatorname{Ker} \omega}(n-4 k, 4 k)$ in the image of the homomorphism

$$
\omega^{!}: \operatorname{Imm}^{\mathbb{Z} / 2<\mathbf{D}_{4}}(n-4 k, 4 k) \rightarrow \operatorname{Imm}^{\operatorname{Ker} \omega}(n-4 k, 4 k) .
$$

Definition 5. Let $\left(f, \Xi_{M}, \kappa\right) \in \operatorname{Imm}^{\mathrm{sf}}(n-k, k)$ be an arbitrary element, where $f: M^{n-k} \rightarrow \mathbb{R}^{n}$ is an immersion of codimension $k$ with the characteristic classes $\kappa \in H^{1}\left(M^{n-k} ; \mathbb{Z} / 2\right)$ of the skew-framing $\Xi_{M}$. We say that the pair $\left(M^{n-k}, \kappa\right)$ admits a retraction of order $q$ if the mapping $\kappa: M^{n-k} \rightarrow \mathbb{R P}^{\infty}$ is represented by the composition $\kappa=I \circ \bar{\kappa}: M^{n-k} \rightarrow \mathbb{R P}^{n-k-q-1} \subset \mathbb{R} \mathrm{P}^{\infty}$. The element $\left[\left(f, \Xi_{M}, \kappa\right)\right]$ admits a retraction of order $q$ if in the cobordism class of this skew-framed immersion, there exists a triple $\left(M^{\prime n-k}, \Xi_{M^{\prime}}, \kappa^{\prime}\right)$ that admits a retraction of order $q$.

Theorem 1. Let $q=q(l)$ be a positive integer, $q=2(\bmod 4)$. Assume that an element $\alpha \in$ $\operatorname{Imm}^{\mathrm{sf}}\left(\frac{3 n+q}{4}, \frac{n-q}{4}\right)$ admits a retraction of order $q$. Then the element $\delta(\alpha) \in \operatorname{Imm}^{\mathbf{D}_{4}}(n-2 k, 2 k), k=\frac{n-q}{4}$, is represented by a $\mathbf{D}_{4}$-framed immersion $\left[\left(g, \Psi_{N}, \eta\right)\right]$ with $\mathbf{I}_{b}$-control.

\section{Proof of Theorem 1}

Denote $n-k-q-1=3 k-1$ by $s$. Let $d: \mathbb{R P}^{s} \rightarrow \mathbb{R}^{n}$ be a generic mapping. We denote the self-intersection points of $d$ (in the target space) by $\Delta(d)$ and the singular points of $d$ by $\Sigma(d)$.

Recall a classification of singular points of generic mappings $\mathbb{R P}^{s} \rightarrow \mathbb{R}^{n}$ in the case $4 s<3 n$. In this range, generic mappings have no quadruple points. Recall the global classification of singularities of generic mappings in this range (for details, see [11]). Singular points (in the target space) are of the following two types:

- a closed manifold $\Sigma^{1,1,0}$

- a singular manifold $\Sigma^{1,0}$ (with singularities of the type $\Sigma^{1,1,0}$ ).

Multiple points are of multiplicities 2 and 3. Triple points form a manifold with boundary and with corners on the boundary. These "corner" singular points on the boundary of the triple-point manifold coincide with the manifold $\Sigma^{1,1,0}$. The regular part of boundary of triple points is a submanifold in $\Sigma^{1,0}$.

Double self-intersection points form a singular submanifold with boundary $\Sigma^{1,0}$. This submanifold is not generic. After an arbitrary small alternation, the double-point manifold becomes a submanifold in $\mathbb{R}^{n}$ with boundary and with corners on the boundary of the type $\Sigma^{1,1,0}$. 
Let $U_{\Sigma}$ be a small regular neighborhood of radius $\varepsilon_{1}$ of the singular submanifold $\Sigma^{1,0}$. Let $U_{\Delta}$ be a small regular neighborhood of the same radius of the submanifold $\Delta(d)$ (this submanifold is immersed with singularities on the boundary). The inclusion $U_{\Sigma} \subset U_{\Delta}$ is well defined.

Consider a regular submanifold in $\Delta$ obtained by cutting a small regular neighborhood of the boundary. This immersed manifold with boundary is denoted by $\Delta^{\text {reg }}$. The (immersed) boundary $\partial \Delta^{\text {reg }}$ is denoted by $\Sigma^{\text {reg }}$. We consider the pair of regular neighborhoods $U_{\Sigma}^{\text {reg }} \subset U_{\Delta}^{\text {reg }}$ of the pair $\Sigma^{\text {reg }} \subset \Delta^{\text {reg }}$ of radius $\varepsilon_{2}, \varepsilon_{2} \ll \varepsilon_{1}$. Since $2 \operatorname{dim}\left(\Delta^{\text {reg }}\right)<n$, after a small perturbation, the manifold $\Delta^{\text {reg }}$ is a submanifold in $U_{\Delta}^{\mathrm{reg}}$.

Let $\left(f_{0}, \Xi_{0}, \kappa\right), f_{0}: M^{n-k} \rightarrow \mathbb{R}^{n}, n-k=\frac{3 n+q}{4}$, be a skew-framed immersion in the cobordism class $\alpha$. We construct an immersion $f: M^{n-k} \rightarrow \mathbb{R}^{n}$ in the regular homotopy class of $f_{0}$ as follows.

Let $\kappa_{0}: M^{n-k} \rightarrow \mathbb{R P}^{s}$ be a retraction of order $q$. Let $f: M \rightarrow \mathbb{R}^{n}$ be an immersion in the regular homotopy class of $f_{0}$ under the condition $\operatorname{dist}\left(d \circ \kappa_{0}, f_{0}\right)<\varepsilon_{3}$. The diameter $\varepsilon_{3}$ of the approximation is given by the inequality $\varepsilon_{3} \ll \varepsilon_{2}$.

Let $g_{1}: N^{n-2 k} \leftrightarrow \mathbb{R}^{n}$ be the immersion parameterizing the double points of $f$. The immersion $g_{1}$ is not generic. After a small perturbation of the immersion $g_{1}$ with the diameter $\varepsilon_{3}$, we obtain a generic immersion $g_{2}: N^{n-2 k} \leftrightarrow \mathbb{R}^{n}$.

The immersed submanifold $g_{2}\left(N^{n-2 k}\right)$ is divided into two submanifolds $g_{2}\left(N_{\text {int }}^{n-2 k}\right)$ and $g_{2}\left(N_{\text {ext }}^{n-2 k}\right)$ with the common boundary $g_{2}\left(\partial N_{\text {int }}^{n-2 k}\right)=g_{2}\left(\partial N_{\text {ext }}^{n-2 k}\right)$ denoted by $g_{2}\left(N_{Q}^{n-2 k-1}\right)$. The manifold $g_{2}\left(N_{\text {int }}^{n-2 k}\right)$ is defined as the intersection of the immersed submanifold $g_{2}\left(N^{n-2 k}\right)$ with the neighborhood $U_{\Delta}^{\text {reg }}$. The manifold $g_{2}\left(N_{\mathrm{ext}}^{n-2 k}\right)$ is defined as the intersection of the immersed submanifold $g_{2}\left(N^{n-2 k}\right)$ with the complement $\mathbb{R}^{n} \backslash\left(U_{\Delta}^{\mathrm{reg}}\right)$. We assume that $g_{2}$ is regular along $\partial U_{\Delta}^{\mathrm{reg}}$. Then $g_{2}\left(N_{Q}^{n-2 k}\right)$ is an immersed submanifold in $\partial U_{\Delta}^{\mathrm{reg}}$. By the construction, the structure group $\mathbf{D}_{4}$ of the normal bundle of the immersed manifold $g_{2}\left(N_{\text {ext }}^{n-2 k}\right)$ admits a reduction to the subgroup $\mathbf{I}_{b} \subset \mathbf{D}_{4}$.

Denote by $L^{n-4 k}$ the self-intersection manifold of the immersion $g_{2}$. This manifold is embedded into $\mathbb{R}^{n}$ by $h: L^{n-4 k} \subset \mathbb{R}^{n}$. The normal bundle of this embedding $h$ is equipped by a $\mathbb{Z} / 2 \imath \mathbf{D}_{4}$-framing denoted by $\Psi_{L}$ and the characteristic class of this framing is denoted by $\zeta_{L}$. By a similar construction, the manifold $L^{n-4 k}$ is decomposed as the union of the two manifolds over a common boundary, denoted by $\Lambda$ : $L^{n-4 k}=L_{\text {ext }}^{n-4 k} \cup_{\Lambda} L_{\mathrm{int}}^{n-4 k}$. The manifold (with boundary) $L_{\mathrm{int}}^{n-4 k}$ is embedded by $h$ into $U_{\Delta}^{\mathrm{reg}}$; the manifold $L_{\text {ext }}^{n-4 k}$ (with the same boundary) is embedded in the complement $\mathbb{R}^{n} \backslash U_{\Delta}^{\text {reg }}$. The common boundary $\Lambda$ is embedded into $\partial U_{\Delta}^{\mathrm{reg}}$.

The manifold $L^{n-4 k}$ is a $\left(\mathbb{Z} / 2 \prec \mathbf{D}_{4}\right)$-framed submanifold in $\mathbb{R}^{n}$. Let us describe the reduction of the structure group of this manifold to the corresponding subgroup in $\mathbb{Z} / 2$ 乙 $\mathbf{D}_{4}$. We describe the subgroups $\mathbf{I}_{2, j}\left(\mathbb{Z} / 2 \oplus \mathbf{D}_{4}\right) \subset \mathbb{Z} / 2 \prec \mathbf{D}_{4}, j=x, y, z$.

Let $a, b$, and $c$ be the generators of the group $\mathbf{D}_{4} \oplus \mathbb{Z} / 2$. To define the subgroups $\mathbf{I}_{2, j} \subset \mathbf{D}_{4}, j=x, y, z$, we describe the transformation of $\mathbb{R}^{4}$ in the standard base $\left(f_{1}, f_{2}, f_{3}, f_{4}\right)$ determined by the generators. Consider the subgroup $\mathbf{I}_{2, x}$. The generator $c_{x}$ (a generator will be equipped with an index corresponding to the subgroup) defines the transformations of the space by the following formulas:

$$
c_{x}\left(f_{1}\right)=f_{3}, \quad c_{x}\left(f_{3}\right)=f_{1}, \quad c_{x}\left(f_{2}\right)=f_{4}, \quad c_{x}\left(f_{4}\right)=f_{2} .
$$

For the generator $a_{x}$ (of order 4), the transformations are as follows:

$$
a_{x}\left(f_{1}\right)=f_{2}, \quad a_{x}\left(f_{2}\right)=-f_{1}, \quad a_{x}\left(f_{3}\right)=f_{4}, \quad a_{x}\left(f_{4}\right)=-f_{3} .
$$

The generator $b_{x}$ (of order 2) defines the transformations of the space by the following formula:

$$
b_{x}\left(f_{1}\right)=f_{2}, \quad b_{x}\left(f_{2}\right)=f_{1}, \quad b_{x}\left(f_{3}\right)=f_{4}, \quad b_{x}\left(f_{4}\right)=f_{3} .
$$

By this formula, the subgroup $\mathbf{D}_{4} \subset \mathbf{D}_{4} \oplus \mathbb{Z} / 2$ is presented by transformations that keep the subspaces $\left(f_{1}, f_{2}\right)$ and $\left.\left(f_{3}, f_{4}\right)\right)$. The generator of the cyclic subgroup $\mathbb{Z} / 2 \subset \mathbf{D}_{4} \oplus \mathbb{Z} / 2$ permutes these planes. 
The subgroups $\mathbf{I}_{2, y}$ and $\mathbf{I}_{2, x}$ are conjugated by the automorphism $O P: \mathbb{Z} / 2 \imath \mathbf{D}_{4} \rightarrow \mathbb{Z} / 2$ 乙 $\mathbf{D}_{4}$ given in the standard base by the formulas

$$
f_{1} \rightarrow f_{1}, \quad f_{2} \rightarrow f_{3}, \quad f_{3} \rightarrow f_{2}, \quad f_{4} \rightarrow f_{4} .
$$

Therefore, the generator $c_{y} \in \mathbf{I}_{2, y}$ is determined by the following transformation:

$$
c_{y}\left(f_{1}\right)=f_{2}, \quad c_{y}\left(f_{2}\right)=f_{1}, \quad c_{y}\left(f_{3}\right)=f_{4}, \quad c_{y}\left(f_{4}\right)=f_{3} .
$$

The generator $a_{y}$ (of order 4 ) is given by

$$
a_{y}\left(f_{1}\right)=f_{3}, \quad a_{y}\left(f_{3}\right)=-f_{1}, \quad a_{y}\left(f_{2}\right)=f_{4}, \quad a_{y}\left(f_{4}\right)=-f_{2} .
$$

The generator $b_{y}$ (of order 2 ) is given by

$$
b_{y}\left(f_{1}\right)=f_{3}, \quad b_{y}\left(f_{3}\right)=f_{1}, \quad b_{y}\left(f_{2}\right)=f_{4}, \quad b_{y}\left(f_{4}\right)=f_{2} .
$$

Let us describe the subgroup $\mathbf{I}_{2, z}$. In this case, the generator $c_{z}$ defines the transformation of the space by the formula $c_{z}\left(f_{i}\right)=-f_{i}, i=1,2,3,4$.

For the generator $a_{z}$ (of order 4 ) the transformation is as follows:

$$
a_{z}\left(f_{1}\right)=f_{2}, \quad a_{z}\left(f_{2}\right)=f_{3}, \quad a_{z}\left(f_{3}\right)=f_{4}, \quad a_{z}\left(f_{4}\right)=f_{1} .
$$

The generator $b_{x}$ (of order 2) defines the transformations of the space by the formulas

$$
b_{z}\left(f_{1}\right)=f_{2}, \quad b_{z}\left(f_{2}\right)=f_{1}, \quad b_{z}\left(f_{3}\right)=f_{4}, \quad b_{z}\left(f_{4}\right)=f_{3} .
$$

Obviously, the restriction of the epimorphism $\omega: \mathbb{Z} / 2 \prec \mathbf{D}_{4} \rightarrow \mathbb{Z} / 2$ to the subgroups $\mathbf{I}_{2, x}, \mathbf{I}_{2, y} \subset \mathbb{Z} / 2 \prec \mathbf{D}_{4}$ is trivial and the restriction of this homomorphism to the subgroup $\mathbf{I}_{2, z}$ is nontrivial.

The subgroup $\mathbf{I}_{3} \subset \mathbf{I}_{2, x}$ is defined as the subgroup with generators $c_{x}, b_{x}$, and $a_{x}^{2}$. This is the index-2 subgroup isomorphic to the group $\mathbb{Z} / 2^{3}$. The image of this subgroup in $\mathbb{Z} / 2 \imath \mathbf{D}_{4}$ coincides with the intersection of an arbitrary pair of subgroups $\mathbf{I}_{2, x}, \mathbf{I}_{2, y}$, and $\mathbf{I}_{2, z}$. The subgroup $\mathbf{I}_{3} \subset \mathbf{I}_{2, y}$ is generated by $c_{y}, b_{y}$, and $a_{y}^{2}$. Moreover, one has $c_{y}=b_{x}, b_{y}=c_{x}$, and $a_{y}^{2}=a_{x}^{2}$. It is easy to verify that the relations $c_{z}=a_{x}^{2}, a_{z}^{2}=c_{x}=b_{y}$, and $b_{z}=b_{x}=c_{y}$ hold. Therefore, $\operatorname{Ker}\left(\left.\omega\right|_{\mathbf{I}_{2}, z}\right)$ coincides with the subgroup $\mathbf{I}_{3} \subset \mathbf{I}_{2, z}$.

The subgroups $\mathbf{I}_{2, x}, \mathbf{I}_{2, y}, \mathbf{I}_{2, z}$, and $\mathbf{I}_{3}$ in $\mathbb{Z} / 2<\mathbf{D}_{4}$ are well defined. There is a natural projection $\pi_{b}: \mathbf{I}_{3} \rightarrow \mathbf{I}_{b}$.

We also consider the subgroup $\mathbf{I}_{2, x \downarrow} \subset \mathbb{Z} / 2 \prec \mathbf{D}_{4}$. This subgroup is a quadratic extension of the subgroup $\mathbf{I}_{2, x}$ such that $\mathbf{I}_{2, x}=\left.\operatorname{Ker} \omega\right|_{\mathbf{I}_{2, x \downarrow}} \subset \mathbf{I}_{2, x \downarrow}$. An explicit definition of this group can be deduced from the geometrical consideration and will not be required.

In the following lemma, we describe the structure group of the framing of the triad $\left(L_{\mathrm{int}}^{n-4 k} \cup_{\Lambda} L_{\mathrm{ext}}^{n-4 k}\right)$. This framing is denoted by $\left(\Psi_{\text {int }} \cup_{\Psi_{\Lambda}} \cup \Psi_{\text {ext }}, \zeta_{\text {int }} \cup_{\zeta_{\Lambda}} \cup \zeta_{\text {ext }}\right)$.

Lemma 1. There exists a generic regular deformation $g_{1} \rightarrow g_{2}$ of diameter $3 \varepsilon_{3}$ such that the immersed manifold $g_{2}\left(N_{\mathrm{ext}}^{n-2 k}\right)$ admits the reduction of the structure group of the $\mathbf{D}_{4}$-framing to the subgroup $\mathbf{I}_{b} \subset \mathbf{D}_{4}$. The manifold $L_{\mathrm{int}}^{n-4 k}$ is divided into the disjoint union of the two manifolds (with boundaries) denoted by $\left(L_{\mathrm{int}, x \downarrow}^{n-4 k}, \Lambda_{x \downarrow}\right)$ and $\left(L_{\mathrm{int}, y}^{n-4 k}, \Lambda_{y}\right)$.

(1) The structure group of the framing $\left(\Psi_{\mathrm{int}, x \downarrow}, \Psi_{\Lambda_{x \downarrow}}\right)$ for the submanifold (with boundary) $\left(L_{\mathrm{int}, x \downarrow}^{n-4 k}, \Lambda_{x \downarrow}\right)$ is reduced to the subgroups $\left(\mathbf{I}_{2, x \downarrow}, \mathbf{I}_{2, z}\right)$. (In particular, the 2 -sheeted cover over $L_{\text {int }, x \downarrow}^{n-4 k}$ classified by $\omega$ (denoted by $\left.\tilde{L}_{\mathrm{int}, x}^{n-4 k} \rightarrow L_{\mathrm{int}, x \downarrow}^{n-4 k}\right)$ is, generally speaking, a nontrivial cover.)

(2) The structure group of the framing $\left(\Psi_{\mathrm{int}, y}, \Psi_{\Lambda}\right)$ for the submanifold (with boundary) $\left(L_{\text {int }, y}^{n-4 k}, \Lambda_{y}\right)$ is reduced to the subgroups $\left(\mathbf{I}_{2, y}, \mathbf{I}_{3}\right)$. (In particular, the 2 -sheeted cover $\tilde{L}_{\mathrm{int}, y}^{n-4 k} \rightarrow L_{\mathrm{int}, y}^{n-4 k}$ classified by $\omega$ is the trivial cover.) Moreover, the double covering $\tilde{L}_{x}^{n-4 k}$ over the component $L_{x \downarrow}^{n-4 k}$ is naturally diffeomorphic to $\tilde{L}_{y}^{n-4 k}$ and this diffeomorphism is in agreement with the restriction of the automorphism $O P: \mathbb{Z} / 2 \imath \mathbf{D}_{4} \rightarrow \mathbb{Z} / 2 \imath \mathbf{D}_{4}$ on the subgroup $\mathbf{I}_{2, x}, \operatorname{OP}\left(\mathbf{I}_{2, x}\right)=\mathbf{I}_{2, y}$. 
(3) The structure group of the framing $\left(\Psi_{\text {ext }}, \zeta_{\text {ext }}\right)$ for the submanifold (with boundary) $h\left(L_{\text {ext }}^{n-4 k}, \Lambda^{n-4 k}\right) \subset\left(\mathbb{R}^{n} \backslash U_{\Delta}^{\mathrm{reg}}, \partial\left(U_{\Delta}^{\mathrm{reg}}\right)\right)$ is reduced to the subgroup $\mathbf{I}_{2, z}$. (In particular, the 2 -sheeted cover $\tilde{L}_{\mathrm{ext}}^{n-4 k} \rightarrow L_{\mathrm{ext}}^{n-4 k}$ classified by $\omega$ is, generally speaking, a nontrivial cover.)

Proof. Components of the self-intersection manifold $g_{1}\left(N^{n-2 k}\right) \backslash\left(g_{1}\left(N^{n-2 k}\right) \cap U_{\Sigma}\right)$ (this manifold is formed by double points $x \in g_{1}\left(N^{n-2 k}\right), x \neq U_{\Sigma}$, with inverse images $\left.\bar{x}_{1}, \bar{x}_{2} \in M^{n-k}\right)$ are classified by the following two types.

Type 1 . The points $\kappa\left(\bar{x}_{1}\right)$ and $\kappa\left(\bar{x}_{2}\right)$ in $\mathbb{R P}^{s}$ are $\varepsilon_{2}$-closed.

Type 2. The distances between the points $\kappa\left(\bar{x}_{1}\right)$ and $\kappa\left(\bar{x}_{2}\right)$ in $\mathbb{R P}^{s}$ are greater than the diameter $\varepsilon_{2}$ of the regular approximation. Points of this type belong to the regular neighborhood $U_{\Delta}$ (of radius $\varepsilon_{1}$ ).

Let us classify components of the triple self-intersection manifold $\Delta_{3}(f)$ of the immersion $f$. A point $x \in \Delta_{3}(f)$ has inverse images $\bar{x}_{1}, \bar{x}_{2}$, and $\bar{x}_{3}$ in $M^{n-k}$. The apriority classification of components is as follows.

Type 1 . The images $\kappa\left(\bar{x}_{1}\right), \kappa\left(\bar{x}_{2}\right)$, and $\kappa\left(\bar{x}_{3}\right)$ are $\varepsilon_{2}$-closed in $\mathbb{R P}^{s}$.

Type 2. The images $\kappa\left(\bar{x}_{1}\right)$ and $\kappa\left(\bar{x}_{2}\right)$ are $\varepsilon_{2}$-closed in $\mathbb{R} \mathrm{P}^{s}$ and the distance between the images $\kappa\left(\bar{x}_{3}\right)$ and $\kappa\left(\bar{x}_{1}\right)$ (or $\left.\kappa\left(\bar{x}_{2}\right)\right)$ is greater than the diameter $\varepsilon_{2}$ of the approximation.

Type 3. The pairwise distances between the points $\kappa\left(\bar{x}_{1}\right), \kappa\left(\bar{x}_{2}\right)$, and $\kappa\left(\bar{x}_{3}\right)$ are greater than the diameter $\varepsilon_{2}$ of the approximation.

By the general-position argument, the component of type 3 does not intersect $d\left(\mathbb{R P}^{s}\right)$. Therefore, the immersion $f$ can be deformed by an $\varepsilon_{2}$-small regular homotopy inside the $\varepsilon_{3}$-regular neighborhood of the regular part of $d\left(\mathbb{R P}^{s}\right)$ such that after this regular homotopy, $\Delta_{3}(f)$ is contained in the complement of $U_{\Delta}^{\text {reg }}$. The codimension of the submanifold $\bar{\Delta}_{2}(d) \subset \mathbb{R} \mathrm{P}^{s}$ is equal to $n-3 k+1=q+k+1$ and is greater than $\operatorname{dim}\left(\Delta_{3}(f)\right)=n-3 k$. By similar arguments, the component of triple points of the type 1 is outside $U_{\Delta}^{\mathrm{reg}}$.

Let us classify components of the quadruple self-intersection manifold $\Delta_{4}(f)$ of the immersion $f$. A point $x \in \Delta_{4}(f)$ has inverse images $\bar{x}_{1}, \bar{x}_{2}, \bar{x}_{3}$, and $\bar{x}_{4}$ in $M^{n-k}$. The apriority classification is as follows.

Type 1. The images $\kappa\left(\bar{x}_{1}\right)$ and $\kappa\left(\bar{x}_{2}\right)$ are $\varepsilon_{2}$-closed in $\mathbb{R} \mathrm{P}^{s}$ and the pairwise distances between the images $\kappa\left(\bar{x}_{1}\right)$ (or $\left.\kappa\left(\bar{x}_{2}\right)\right), \kappa\left(\bar{x}_{3}\right)$, and $\kappa\left(\bar{x}_{4}\right)$ are sufficiently large with respect to the diameter $\varepsilon_{2}$ of the approximation.

Type 2. The two pairs $\left(\kappa\left(\bar{x}_{1}\right), \kappa\left(\bar{x}_{2}\right)\right)$ and $\left(\kappa\left(\bar{x}_{3}\right), \kappa\left(\bar{x}_{4}\right)\right)$ of images are $\varepsilon_{2}$-closed in $\mathbb{R P}^{s}$ and the distance between the images $\kappa\left(\bar{x}_{1}\right)$ (or $\left.\kappa\left(\bar{x}_{2}\right)\right)$ and $\kappa\left(\bar{x}_{3}\right)$ (or $\left.\kappa\left(\bar{x}_{4}\right)\right)$ is sufficiently large with respect to the diameter $\varepsilon_{2}$ of the approximation. (The described component is the complement of the regular $\varepsilon_{2}$-neighborhood of the triple-point manifold of $d\left(\mathbb{R P}^{s}\right)$.)

Type 3. The images $\kappa\left(\bar{x}_{1}\right), \kappa\left(\bar{x}_{2}\right)$, and $\kappa\left(\bar{x}_{3}\right)$ on $\mathbb{R P}^{s}$ are pairwise $\varepsilon_{2}$-closed in $\mathbb{R P}^{s}$ and the distance between the images $\kappa\left(\bar{x}_{1}\right)$ (or $\kappa\left(\bar{x}_{2}\right)$, or $\kappa\left(\bar{x}_{3}\right)$ ) and $\kappa\left(\bar{x}_{4}\right)$ is sufficiently large with respect to the diameter $\varepsilon_{2}$ of the approximation.

Type 4. All the images $\kappa\left(\bar{x}_{1}\right), \kappa\left(\bar{x}_{2}\right), \kappa\left(\bar{x}_{3}\right)$, and $\kappa\left(\bar{x}_{4}\right)$ are pairwise $\varepsilon_{2}$-closed in $\mathbb{R} \mathrm{P}^{s}$.

Let us prove that there exists a generic $f$ such that the components of type 1 and type 3 are empty. For the component of type 3 , the proof is similar to the proof for the component of type 1 .

Let us prove that there exists a generic deformation $g_{1} \rightarrow g_{2}$ with diameter $3 \varepsilon_{3}$ such that after this deformation in the neighborhood $U_{\Delta}^{\text {reg }}$, there are no self-intersection points of $g_{2}$ obtained by a generic resolution of triple points of $f$ of the types 1 and 2. Let us start with the proof for triple points of the type 1.

For a generic small alternation of the immersion $g_{2}$ inside $U_{\Delta}^{\text {reg }}$, the points of type 1 of the triple-point manifold $\Delta_{3}(f)$ are perturbed into a component of the self-intersection points on $L^{n-4 k}$. This component is classified by the following two subtypes:

- Subtype (a). Preimages of a point is $\left(\bar{x}_{2}, \bar{x}_{1}\right),\left(\bar{x}_{2}, \bar{x}_{1}^{\prime}\right)$.

- Subtype (b). Preimages of a point is $\left(\bar{x}_{1}, \bar{x}_{1}^{\prime}\right),\left(\bar{x}_{1}, \bar{x}_{2}\right)$. 
In this formula, the points with common indices have $\varepsilon_{3}$-closed projections on the corresponding sheet of $d\left(\mathbb{R P}^{s}\right)$. The two points in a pair form a point on $N^{n-2 k}$ and a couple of pairs forms a point on the component of $L^{n-4 k}$.

Let us prove that there exists a $2 \varepsilon_{3}$-small regular deformation $g_{1} \rightarrow g_{2}$ such that the component of $h\left(L^{n-4 k}\right) \cap U_{\Delta}^{\text {reg }}$ of the subtype (a) is empty. Let $K^{s-k}$ be the intersection manifold of $f\left(M^{n-k}\right)$ with $d\left(\mathbb{R P}^{s}\right)$ (immersed into $\left.\mathbb{R P}^{s}\right)$. By general-position arguments, since $2 s<n-2 k$, a generic perturbation of $K^{s-k} \rightarrow \mathbb{R P}^{s}$ in $\mathbb{R}^{n}$ is an embedded submanifold. Therefore, there exists a $2 \varepsilon_{2}$-small deformation of $K^{s-k}$ in $\mathbb{R}^{n}$ into $K^{s-k}$ such that the $\varepsilon_{2}$-neighborhood of $K^{s-k}$ has no self-intersections. The deformation $K^{s-k} \rightarrow K^{s-k}$ is extended to the deformation of $g_{1}\left(N^{n-2 k}\right)$ in a neighborhood of the considered component. After the described deformation, $g_{2}\left(N^{n-2 k}\right)$ has no self-intersection components of the subtype (a). The case of self-intersection of the subtype (b) is similar.

Let us describe a generic deformation $g_{1} \rightarrow g_{2}$ with the support in $U_{\Delta}^{\text {reg }}$ that resolves quadruple points of $f$ of the type 2. This deformation could be arbitrarily small. After the deformation, the component $\Delta_{4}(f)$ of the type 2 determines two components of $L^{n-4 k}$ of different subtypes. These two components are denoted by $L_{x}^{n-4 k}$ and $L_{y}^{n-4 k}$.

The immersed submanifold $g_{2}\left(N^{n-2 k}\right) \cap U_{\Delta}^{\text {reg }}$ is divided into two components. The first component is formed by pairs of points $\left(\bar{x}, \bar{x}^{\prime}\right)$ with the $3 \varepsilon_{3}$-closed images $\kappa(\bar{x}), \kappa\left(\bar{x}^{\prime}\right)$ on $\mathbb{R} \mathrm{P}^{s}$. This component is denoted by $g_{2}\left(N_{x}^{n-2 k}\right)$. The last component of $g_{2}\left(N^{n-2 k}\right) \cap U_{\Delta}^{\text {reg }}$ is denoted by $g_{2}\left(N_{y}^{n-2 k}\right)$. This component is formed by pairs of points $\left(\bar{x}, \bar{x}^{\prime}\right)$ with the projections $\left(\kappa(\bar{x}), \kappa\left(\bar{x}^{\prime}\right)\right)$ on different sheets of $\mathbb{R} \mathrm{P}^{s}$.

The component $L_{x \downarrow}^{n-4 k}$ is defined by pairs $\left(\bar{x}_{1}, \bar{x}_{1}^{\prime}\right)$ and $\left(\bar{x}_{2}, \bar{x}_{2}^{\prime}\right)$. The component $L_{y}^{n-4 k}$ is defined by pairs $\left(\bar{x}_{1}, \bar{x}_{2}\right)$ and $\left(\bar{x}_{1}^{\prime}, \bar{x}_{2}^{\prime}\right)$. A common index of points in the pair means that the images of the points are $\varepsilon_{3}$-closed on $\mathbb{R P}^{s}$. Each pair consists of the first two points and the second two points. Each two points of this pair determine two points on $N^{n-2 k}$ respectively with the same image of $g_{2}$. It is easy to see that the component $L_{x \downarrow}^{n-4 k}$ is the self-intersection of $g_{2}\left(N_{x}^{n-2 k}\right)$ and the component $L_{y}^{n-4 k}$ is the self-intersection of $g_{2}\left(N_{y}^{n-2 k}\right)$.

It is easy to see that the structure groups of the components are in agreement with the corresponding subgroup described in the lemma. The component $L_{x \downarrow}^{n-4 k}$ admits the reduction of the structure group to the subgroup $\mathbf{I}_{2, x \downarrow} \subset \mathbb{Z} / 2 \prec \mathbf{D}_{4}$. The component $L_{y}^{n-4 k}$ admits a reduction of the structure group to the subgroup $\mathbf{I}_{2, y}$. Moreover, it is easy to see that the covering $\tilde{L}_{x \downarrow}^{n-4 k}$ over $L_{x}^{n-4 k}$ induced by the epimorphism $\omega: \mathbb{Z} / 2 \imath \mathbf{D}_{4} \rightarrow \mathbb{Z} / 2$ with the kernel $\mathbf{I}_{2, x} \subset \mathbb{Z} / 2 \imath \mathbf{D}_{4}$ is naturally diffeomorphic to $L_{y}^{n-4 k}$. Also, it is easy to see that this diffeomorphism is in agreement with the transformation $O P$ of the structure groups of the framing over the components.

The last component of $L^{n-4 k}$ is immersed in an $\varepsilon_{2}$-neighborhood of $d\left(\mathbb{R P}^{s}\right)$ outside of $U_{\Delta}^{\text {reg }}$ and is denoted by $L_{z}^{n-4 k}$. The structure group of the framing of this component is $\mathbf{I}_{2, z}$. Lemma 1 is proved.

The last part of the proof of the Theorem 1. Let us construct a pair of polyhedra

$$
\left(P^{\prime}, Q^{\prime}\right) \subset \mathbb{R}^{n}, \quad \operatorname{dim}\left(P^{\prime}\right)=2 s-n=n-2 k-q-2, \quad \operatorname{dim}\left(Q^{\prime}\right)=\operatorname{dim}\left(P^{\prime}\right)-1 .
$$

Obviously, $\operatorname{dim}\left(P^{\prime}\right)<2 k-1$. Take a generic mapping $d^{\prime}: \mathbb{R P}^{s} \rightarrow \mathbb{R}^{n}$. Let us consider the submani-

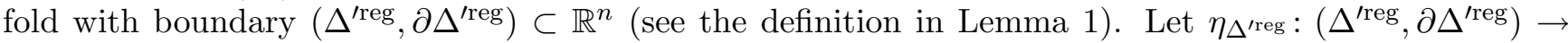
$\left(K\left(\mathbf{D}_{4}, 1\right), K\left(\mathbf{I}_{b}, 1\right)\right)$ be the classifying mapping for the double-point self-intersection manifold of $d$.

By the standard argument, we can take a mapping $d$ such that the mapping $\eta_{\Delta^{\text {reg }}}$ is a homotopy equivalence of the pairs up to dimension $q+1$. After this modification $d^{\prime} \rightarrow d$, we define $(P, Q)=$ $\left(\Delta^{\text {reg }}, \partial \Delta^{\text {reg }}\right) \subset \mathbb{R}^{n}$, and the mapping $\eta_{\Delta^{\text {reg }}}$ is a $(q+1)$-homotopy equivalence.

The subpolyhedron $Q$ is equipped with two cohomological classes $\kappa_{Q, 1}, \kappa_{Q, 2} \in H^{1}(Q ; \mathbb{Z} / 2)$. Since $\Sigma$ is the submanifold in $\mathbb{R P}^{s}$, the restriction of the canonical class $\kappa \in H^{1}\left(\mathbb{R} \mathrm{P}^{s} ; \mathbb{Z} / 2\right)$ on $H^{1}(\Sigma ; \mathbb{Z} / 2)$ is well defined. The inclusion $i_{Q}: Q \subset U_{\Sigma}$ determines the cohomological class $\left(i_{Q}\right)^{*}(\kappa) \in H^{1}(Q ; \mathbb{Z} / 2)$. The cohomological class $\kappa_{Q, 1}$ is defined as the characteristic class of the canonical double-points covering over $\Sigma$. The class $\kappa_{Q, 2}$ is defined by the formula $\kappa_{Q, 2}=\left(i_{Q}\right)^{*}(\kappa)+\kappa_{Q, 1}$. 
The immersed manifold (with boundary) $\left(N^{n-2 k} \cap U_{\Sigma}\right) \rightarrow U_{\Sigma}$ is equipped with an $\mathbf{I}_{b}$-framing. Obviously, the classes $\kappa_{Q, 1}, \kappa_{Q, 2} \in H^{1}\left(U_{\Sigma} ; \mathbb{Z} / 2\right)=H^{1}(Q ; \mathbb{Z} / 2)$ restricted on $H^{1}\left(g_{2}\left(N_{\text {ext }}^{n-2 k}\right) ; \mathbb{Z} / 2\right)$ (recall that $\left.g_{2}\left(N_{\text {ext }}^{n-2 k}\right)=g_{2}\left(N^{n-2 k}\right) \cap\left(\mathbb{R}^{n} \backslash U_{\Delta}\right)\right)$ are in agreement with the two generated cohomological classes $\rho_{1}$ and $\rho_{2}$ of the $\mathbf{I}_{b}$-framing, respectively.

Let us define the immersion $g: N^{n-2 k} \rightarrow \mathbb{R}^{n}$ with $\mathbf{I}_{b}$-control over $(P, Q)$. Let us start with the immersion $g_{2}: N^{n-2 k} \rightarrow \mathbb{R}^{n}$ constructed in Lemma 1. By a $2 \varepsilon_{2}$-small generic regular deformation, we can deform the immersion $g_{2}$ into $g_{3}$ such that this deformation pushes the component $g_{2}\left(N_{x}^{n-2 k}\right)$ out of $U_{\Delta}^{\text {reg }}$. Therefore, the component $L_{x \downarrow}^{n-4 k} \subset L^{n-4 k}$ of the self-intersection of $g_{2}$ is also deformed out of $U_{\Delta}^{\text {reg }}$.

The immersed manifold (with boundary) $g_{3}\left(N^{n-2 k}\right) \cap\left(\mathbb{R}^{n} \backslash U_{\Delta}^{\text {reg }}\right)$ is equipped with the $\mathbf{I}_{b^{-}}$framing of the normal bundle. Obviously, the classes $\kappa_{Q, 1}, \kappa_{Q, 2} \in H^{1}\left(U_{\Sigma} ; \mathbb{Z} / 2\right)=H^{1}(Q ; \mathbb{Z} / 2)$ restricted on $H^{1}\left(g_{2}\left(N^{n-2 k}\right) \cap U_{\Delta} ; \mathbb{Z} / 2\right)$ agree with the two generated cohomological classes of the $\mathbf{I}_{b}$-framing. The immersed manifold $g_{3}\left(N^{n-2 k}\right) \cap U_{\Delta}^{\text {reg }}$ coincides with $g_{2}\left(N_{y}^{n-2 k}\right)$ and has the general structure group of the framing. This immersed manifold has the self-intersection manifold (with boundary) $h\left(L^{n-4 k}\right) \cap U_{\Delta}^{\text {reg }}$ with the reduction of the structure group to the pair of subgroups $\left(\mathbf{I}_{2, y}, \mathbf{I}_{3}\right)$.

Let us prove that the immersed manifold (with boundary) $h\left(L^{n-4 k}\right) \cap U_{\Delta}^{\text {reg }}$ is $\left(\mathbb{Z} / 2 \imath \mathbf{D}_{4}\right)$-framed cobordant (relative to the boundary) to a $\left(\mathbb{Z} / 2 \imath \mathbf{D}_{4}\right)$-framed manifold decomposed into the disjoint union of a closed $\left(\mathbb{Z} / 2<\mathbf{D}_{4}\right)$-framed manifold that is the image of the transfer homomorphism $\omega^{!}$and a relative $\mathbf{I}_{3}$-framed manifold.

Take a $\left(\mathbb{Z} / 2 \prec \mathbf{D}_{4}\right)$-framed manifold $\left(\tilde{L}^{n-4 k}, \tilde{\Psi}, \tilde{\zeta}\right)$ that is defined as the image of the $\left(\mathbb{Z} / 2 \imath \mathbf{D}_{4}\right)$-framed manifold $\left(L^{n-4 k}, \Psi, \zeta\right)$ by the transfer homomorphism (a double covering) with respect to the cohomological class $\omega \in H^{1}\left(\mathbb{Z} / 2 \nmid \mathbf{D}_{4} ; \mathbb{Z} / 2\right)$. Recall that the manifold $\tilde{L}^{n-4 k}$ is obtained by gluing the manifolds $\tilde{L}_{x}^{n-4 k} \cup \tilde{L}_{y}^{n-4 k}$ with the manifold $\tilde{L}_{z}^{n-4 k}$ along the common boundary $\tilde{\Lambda}^{n-4 k-1}$. Note that the group of the framing of the last manifold $\tilde{\Lambda}_{z}^{n-4 k-1}$ is the subgroup $\mathbf{I}_{3} \subset \mathbb{Z} / 2 \prec \mathbf{D}_{4}$.

Let $O P \alpha$ be the $\left(\mathbb{Z} / 2<\mathbf{D}_{4}\right)$-framed immersion obtained from an arbitrary $\left(\mathbb{Z} / 2 \prec \mathbf{D}_{4}\right)$-framed immersion $\alpha$ by changing the structure group of the framing by the transformation $O P$. The $\left(\mathbb{Z} / 2 \imath \mathbf{D}_{4}\right)$-framed manifold (with boundary) $\left(\tilde{L}_{y}^{n-4 k}, \tilde{\Psi}_{y}, \tilde{\zeta}_{y}\right)$ coincides with the two disjoint copies of the $\left(\mathbb{Z} / 2 \imath \mathbf{D}_{4}\right)$-framed manifold (with boundary) $O P\left(\tilde{L}_{y}^{n-4 k}, \tilde{\Psi}_{y}, \tilde{\zeta}_{y}\right)$.

Let

$$
\alpha_{1}=-O P\left(\tilde{L}^{n-4 k}, \tilde{\Psi}, \tilde{\zeta}\right) .
$$

Define the sequence of $\left(\mathbb{Z} / 2\right.$ 々 $\left.\mathbf{D}_{4}\right)$-framed immersions

$$
\alpha_{2}=-2 O P \alpha_{1}, \quad \alpha_{3}=-2 O P \alpha_{2}, \ldots, \quad \alpha_{j}=-2 O P \alpha_{j-1} .
$$

Obviously, the $\left(\mathbf{D} / 4\right.$ 乙Z/2)-framed immersion $\alpha_{1}+\alpha_{2}=\alpha_{1}+2 O P \alpha_{1}^{-1}$ is represented by three copies of the manifold $\tilde{L}^{n-4 k}$. The second and third copies are obtained from the first copy by a change in the orientation and a change in the structure group of the framing. The manifold $-O P\left[\tilde{L}^{n-4 k}\right] \cup 2\left[\tilde{L}^{n-4 k}\right]$ contains, in particular, a copy of $-O P\left[\tilde{L}_{x}^{n-4 k}\right]$ inside the first component and the union $\left[\tilde{L}_{y}^{n-4 k} \cup L_{y}^{n-4 k}\right]$ of the mirror two copies of $-O P\left[\tilde{L}_{x}^{n-4 k}\right]$ in the second and third components. Therefore, the manifold $-O P\left[\tilde{L}^{n-4 k}\right] \cup 2\left[\tilde{L}^{n-4 k}\right]$ is $\left(\mathbb{Z} / 2\left\langle\mathbf{D}_{4}\right)\right.$-framed cobordant to the $\left(\mathbb{Z} / 2\left\langle\mathbf{D}_{4}\right)\right.$-framed manifold obtained by gluing the union of a copy of $-O P\left[\tilde{L}_{x}^{n-4 k}\right]$ and four copies of $\tilde{L}_{y}^{n-4 k}$ by an $\mathbf{I}_{3}$-framing manifold along the boundary. This cobordism is relative with respect to the submanifold $-O P\left[\tilde{L}_{z}^{n-4 k} j_{\phi} \cup 2\left[L_{z}^{n-4 k}\right] \subset-O P\left[L^{n-4 k}\right] \cup 2 L^{n-4 k}\right.$.

By a similar argument, it is easy to prove that the element $\aleph=\sum_{j=1} \alpha_{j}$ is $\left(\mathbb{Z} / 2 \prec \mathbf{D}_{4}\right)$-framed cobordant to the manifold obtained by gluing the union $-O P\left[\tilde{L}_{x}^{n-4 k}\right] \cup 2^{j}(-O P)^{j-1}\left[\tilde{L}_{y}^{n-4 k}\right]$ by an $\mathbf{I}_{3}$-manifold along the boundary. Moreover, this cobordism is relative with respect to all copies of $\tilde{L}_{z}^{n-4 k}$ (with various orientations). If $j_{0}$ is sufficiently large, the manifold (with $\mathbf{I}_{3}$-framed boundary) $2^{j}(-O P)^{j_{0}-1}\left[\tilde{L}_{y}^{n-4 k}\right]$ is cobordant with respect to the boundary to an $\mathbf{I}_{3}$-framed manifold.

Therefore, the manifold $L_{y}^{n-4 k}$ is $\left(\mathbb{Z} / 2 \prec \mathbf{D}_{4}\right)$-framed cobordant with respect to the boundary to the union of an $\mathbf{I}_{3}$-framed manifold with the same boundary and a closed manifold that is the double cover 
with respect to $\omega$ over a $\left(\mathbb{Z} / 2 \succ \mathbf{D}_{4}\right)$-framed manifold. This cobordism is realized as a cobordism of the self-intersection of a $\mathbf{D}_{4}$-framed cobordism with support inside $U_{\Delta}^{\text {reg }}$ from the immersion $g_{3}$ to a $\mathbf{D}_{4}$-framed immersion $g_{4}$. After an additional deformation of $g_{4}$ inside a larger neighborhood of $\Delta^{\text {reg }}$, the relative $\mathbf{I}_{b}$-submanifold of the self-intersection manifold of $g_{4}$ is deformed outside of $U_{\Delta}^{\text {reg }}$. The $\mathbf{D}_{4}$-framed immersion obtained as a result of this cobordism admits an $\mathbf{I}_{b}$-control. Theorem 1 is proved.

\section{4. $(\mathbb{Z} / 2 \oplus \mathbb{Z} / 4)$-Structure (Cyclic Structure) on the Self-Intersection of a $\mathrm{D}_{4}$-Framed Immersion}

Let us describe the subgroup $\mathbf{I}_{4} \subset \mathbb{Z} / 2 \succ \mathbf{D}_{4}$. This subgroup is isomorphic to the group $\mathbb{Z} / 2 \oplus \mathbb{Z} / 4$. Recall that the group $\mathbb{Z} / 2 \imath \mathbf{D}_{4}$ is the transformation group of $\mathbb{R}^{4}$ that keeps the two planes $\left(f_{1}, f_{2}\right)$ and $\left(f_{3}, f_{4}\right)$ spanned by the vectors of the standard base $\left(f_{1}, f_{2}, f_{3}, f_{4}\right)$ (the planes can be kept fixed or be permuted by means of a transformation).

Denote the generators of $\mathbb{Z} / 2 \oplus \mathbb{Z} / 4$ by $l$ and $r$. Describe the transformations of $\mathbb{R}^{4}$ given by each generator. Consider a new base $\left(e_{1}, e_{2}, e_{3}, e_{4}\right)$ given by

$$
e_{1}=f_{1}+f_{2}, \quad e_{2}=f_{1}-f_{2}, \quad e_{3}=f_{3}+f_{4}, \quad e_{4}=f_{3}-f_{4} .
$$

The generator $r$ of order 4 is represented by the rotation in the plane $\left(e_{2}, e_{4}\right)$ by the angle $\frac{\pi}{2}$ and the reflection in the plane $\left(e_{1}, e_{3}\right)$ with respect to the line $e_{1}+e_{3}$. The generator $l$ of order 2 is represented by the central symmetry in the plane $\left(e_{1}, e_{3}\right)$ (or, equivalently, in the plane $\left(f_{1}, f_{3}\right)$ ).

Obviously, the described representation of $\mathbf{I}_{4}$ admits the invariant $(1,1,2)$-dimensional subspaces. We denote these subspaces by $\lambda_{1}, \lambda_{2}$, and $\tau$.

The line subspaces $\lambda_{1}$ and $\lambda_{2}$ are generated by the vectors $e_{1}+e_{3}$ and $e_{1}-e_{3}$, respectively. The subspace $\tau$ is generated by the vectors $e_{2}$ and $e_{4}$. The generator $r$ acts by the reflection in $\kappa_{2}$ and by the rotation in $\tau$ by the angle $\frac{\pi}{2}$. The generator $l$ acts by reflections in the subspaces $\lambda_{1}$ and $\lambda_{2}$. In particular, if the structure group $\mathbb{Z} / 2 \imath \mathbf{D}_{4}$ of a 4-dimensional bundle $\zeta: E(\zeta) \rightarrow L$ admits the reduction to the subgroup $\mathbf{I}_{4}$, then the bundle is decomposed into the direct $\operatorname{sum} \zeta=\lambda_{1} \oplus \lambda_{2} \oplus \tau$ of 1-, 1-, and 2-dimensional subbundles.

Definition 6. Let $\left(g: N^{n-2 k} \leftrightarrow \mathbb{R}^{n}, \Xi_{N}, \eta\right)$ be an arbitrary $\mathbf{D}_{4}$-framed immersion. We say that this immersion is a cyclic immersion if the structure group $\mathbb{Z} / 2 \prec \mathbf{D}_{4}$ of the normal bundle over the double-point manifold $L^{n-4 k}$ of this immersion admits a reduction to the subgroup $\mathbf{I}_{4} \subset \mathbb{Z} / 2 \prec \mathbf{D}_{4}$. In this definition, we assume that the pairs $\left(f_{1}, f_{2}\right)$ and $\left(f_{3}, f_{4}\right)$ are the vectors of the framing for the two sheets of the canonical covering $\bar{L}^{n-4 k}$ over the double-point manifolds $L^{n-4 k}$.

In particular, for a cyclic $\left(\mathbb{Z} / 2 \succ \mathbf{D}_{4}\right)$-framed immersion, there exist mappings $\kappa_{a}: L^{n-4 k} \rightarrow K(\mathbb{Z} / 2,1)$ and $\mu_{a}: L^{n-4 k} \rightarrow K(\mathbb{Z} / 4,1)$ such that the characteristic mapping $\zeta: L^{n-4 k} \rightarrow K(\mathbb{Z} / 2 \imath \mathbf{D} / 4,1)$ of the $\left(\mathbb{Z} / 2 \prec \mathbf{D}_{4}\right)$-framing of the normal bundle over $L^{n-4 k}$ reduces to the mapping with the image $K\left(\mathbf{I}_{b}, 1\right)$ and the equation $\zeta=i\left(\kappa_{a} \oplus \mu_{a}\right)$ holds, where $i: \mathbb{Z} / 2 \oplus \mathbb{Z} / 4 \rightarrow \mathbf{I}_{4}$ is the prescribed isomorphism.

The following proposition is proved by a straightforward calculation.

Proposition 1. Let $\left(g, \Psi_{N}, \eta\right)$ be a $\mathbf{D}_{4}$-framed immersion that is a cyclic immersion. Then the Kervaire invariant included in the diagram (4) can be calculated by the formula

$$
\Theta_{a}=\left\langle\kappa_{a}^{\frac{n-4 k}{2}} \mu_{a}^{*}(\tau)^{\frac{n-4 k-2}{4}} \mu_{a}^{*}(\rho) ;[L]\right\rangle,
$$

where $\tau \in H^{2}(\mathbb{Z} / 4 ; \mathbb{Z} / 2)$ and $\rho \in H^{1}(\mathbb{Z} / 4 ; \mathbb{Z} / 2)$ are the generators.

Proof. Consider the subgroup of index $2 \mathbf{I}_{b} \subset \mathbf{I}_{4}$. This subgroup is the kernel of the epimorphism $\chi^{\prime}: \mathbf{I}_{4} \rightarrow \mathbb{Z} / 2$, which is the restriction of the characteristic class $\chi: \mathbb{Z} / 2$ ? $\mathbf{D}_{4} \rightarrow \mathbb{Z} / 2$ of the canonical double cover $\bar{L} \rightarrow L$ to the subgroup $\mathbf{I}_{4} \subset \mathbb{Z} / 2 \imath \mathbf{D}_{4}$. Obviously, the characteristic class (5) is calculated by the formula

$$
\Theta_{a}=\left\langle\hat{\kappa}_{a}^{\frac{n-4 k}{2}} \hat{\rho}_{a}^{\frac{n-4 k}{2}} ; \bar{L}\right\rangle
$$


where the characteristic class $\hat{\kappa}_{a} \in H^{1}(\bar{L} ; \mathbb{Z} / 2)$ is induced from the class $\kappa_{a} \in H^{1}(L ; \mathbb{Z} / 2)$ by the canonical cover $\bar{L} \rightarrow L$, and the class $\hat{\rho}_{a} \in H^{1}(\bar{L} ; \mathbb{Z} / 2)$ is obtained by the transfer of the class $\rho \in H^{1}(L ; \mathbb{Z} / 4)$.

Note that $\hat{\kappa}_{a}=\tau_{1}$ and $\hat{\rho}_{a}=\tau_{2}$, where $\tau_{1}$ and $\tau_{2}$ are the two $\mathbf{I}_{b}$-characteristic classes. Therefore, $\hat{\kappa}_{a} \hat{\rho}_{a}=\tau_{1} \tau_{2}=w_{2}(\eta)$, where $\eta$ is the two-dimensional bundle that determines the $\mathbf{D}_{4}$-framing (over the submanifold $\bar{L}^{n-4 k} \subset N^{n-2 k}$, this framing admits the reduction to an $\mathbf{I}_{b}$-framing) of the normal bundle for the immersion $g\left(N^{n-2 k}\right)$.

Therefore, the characteristic number given by formula (5) in the case where the $\left(\mathbb{Z} / 2\right.$ 乙 $\left.\mathbf{D}_{4}\right)$-framing over $L^{n-4 k}$ is reduced to the $\mathbf{I}_{4}$-framing, coincides with the characteristic number given by formula (6). Proposition 1 is proved.

Definition 7. We say that a $\mathbf{D}_{4}$-framed immersion $\left(g, \Xi_{N}, \eta\right)$ admits a $(\mathbb{Z} / 2 \oplus \mathbb{Z} / 4)$-structure if for the double-point manifold $L^{n-4 k}$ of $g$, there exist mappings $\kappa_{a}: L^{n-4 k} \rightarrow K(\mathbb{Z} / 2,1)$ and $\mu_{a}: L^{n-4 k} \rightarrow$ $K(\mathbb{Z} / 4,1)$ such that the characteristic number (5) coincides with the Kervaire invariant (see Definition 2).

Theorem 2. Let $(g, \Xi, \eta)$ be a $\mathbf{D}_{4}$-framed immersion, $g: N^{n-2 k} \rightarrow \mathbb{R}^{n}$, that represents a regular cobordism class in $\operatorname{Im}\left(\delta: \operatorname{Imm}^{\mathrm{sf}}(n-k, k) \rightarrow \operatorname{Imm}^{\mathbf{D}_{4}}(n-2 k, 2 k)\right), n-4 k=62, n=2^{l}-2, l \geq 15$, and the conditions of Theorem 1 hold, i.e., the residue class $\delta^{-1}\left(\operatorname{Imm}^{\mathrm{sf}}(n-k, k)\right)$ (this class is defined modulo odd torsion) contains a $\mathbf{D}_{4}$-framed immersion that admits a retraction of order 62 . Then in the skew-framed cobordism class $(g, \Xi, \eta)=\delta[(f, \Xi, \kappa)] \in \operatorname{Imm}^{\mathrm{sf}}(n-k, k)$, there exists a skew-framed immersion that admits $a(\mathbb{Z} / 2 \oplus \mathbb{Z} / 4)$-structure.

\section{Proof of Theorem 2}

Let us state the geometric control principle for $\mathbf{I}_{b}$-controlled immersions.

Let us take an $\mathbf{I}_{b}$-controlled immersion $\left(g, \Xi_{N}, \eta ;(P, Q), \kappa_{Q, 1}, \kappa_{Q, 2}\right)$, where $g: N \uparrow \mathbb{R}^{n}$ is a $\mathbf{D}_{4}$-framed immersion equipped by a control mapping over a polyhedron $i_{P}: P \subset \mathbb{R}^{n}, \operatorname{dim}(P)=2 k-1, Q \subset P$, $\operatorname{dim}(Q)=\operatorname{dim}(P)-1$. The characteristic classes $\kappa_{Q, i} \in H^{1}(Q ; \mathbb{Z} / 2), i=1,2$, coincide with characteristic classes $\kappa_{1} \in N_{\text {int }}^{n-2 k}$ by means of the mapping $\partial N_{\text {int }}^{n-2 k}=N_{Q}^{n-2 k} \rightarrow Q$, where $N_{\text {int }}^{n-2 k} \subset N^{n-2 k}, N_{\text {int }}^{n-2 k}=$ $g^{-1}\left(U_{P}\right), U_{P} \subset \mathbb{R}^{n}$.

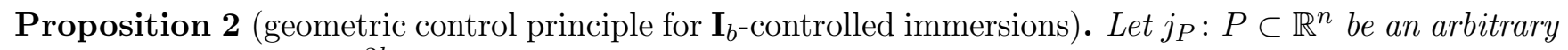
embedding and $g_{1}: N^{n-2 k} \rightarrow \mathbb{R}^{n}$ be an arbitrary mapping such that the restriction

$$
\left.g_{1}\right|_{N_{\mathrm{int}}}:\left(N_{\mathrm{int}}^{n-2 k}, N_{Q}^{n-2 k-1}\right) \rightarrow\left(U_{P}, \partial U_{P}\right)
$$

is an immersion (the restriction $\left.g\right|_{N_{Q}^{n-2 k-1}}$ is an embedding) that corresponds to the immersion

$$
\left.g\right|_{N_{\text {int }}^{n-2 k}}:\left(N_{\text {int }}^{n-2 k}, N_{Q}^{n-2 k-1}\right) \rightarrow\left(U_{P}, \partial U_{P}\right)
$$

by means of the standard diffeomorphism of regular neighborhoods $U_{i_{P}}=U_{j_{P}}$ of subpolyhedra $i(P)$ and $j(P)$. (Since $2 \operatorname{dim}(P)<n-1$, there is only one diffeomorphism of $U_{i_{P}}$ and $U_{j_{P}}$ up to an isotopy.) Then for an arbitrary $\varepsilon>0$, there exists an immersion $g_{\varepsilon}: N^{n-2 k} \rightarrow \mathbb{R}^{n}$ such that $\operatorname{dist}_{C^{0}}\left(g_{1}, g_{\varepsilon}\right)<\varepsilon$ and, in addition, $g_{\varepsilon}$ is regular homotopy to an immersion $g$ and the restrictions $\left.g_{\varepsilon}\right|_{N_{\mathrm{int}}^{n-2 k}}$ and $\left.g_{1}\right|_{N_{\mathrm{int}}^{n-2 k}}$ coincide.

We start the proof of Theorem 2 with the following construction. Let us consider the manifold $Z=S^{\frac{n}{2}+64} / i \times \mathbb{R} \mathrm{P}^{\frac{n}{2}+64}$. This manifold is the direct product of two standard lens spaces $(\bmod 4)$. The cover $p_{Z}: \hat{Z} \rightarrow Z$ over this manifold with the covering space $\hat{Z}=\mathbb{R P}^{\frac{n}{2}+64} \times \mathbb{R P}^{\frac{n}{2}+64}$ is well defined.

Let us consider in the manifold $Z$ a family of submanifolds $X_{i}, i=0, \ldots, \frac{n+2}{128}$, of codimension $\frac{n+2}{2}$, defined by the formula

$$
\begin{aligned}
X_{0}=S^{\frac{n}{2}+64} / i \times \mathbb{R} P^{63}, \quad X_{1}=S^{\frac{n}{2}+32} / i \times \mathbb{R} \mathrm{P}^{95}, \quad \ldots, \\
X_{j}=S^{\frac{n}{2}-32(j-2)-1} / i \times \mathbb{R} \mathrm{P}^{32(j+2)-1}, \quad \ldots, \quad X_{\frac{n+2}{64}}=S^{63} / i \times \mathbb{R P}^{\frac{n}{2}+64} .
\end{aligned}
$$


The embedding of the corresponding manifold in $Z$ is defined by the Cartesian product of two standard embeddings.

The union of submanifolds $\left\{X_{i}\right\}$ is the stratified submanifold (with singularities) $X \subset Z$ of dimension $\frac{n}{2}+127$; the codimension of maximal singular strata in $X$ is equal to 64 . The covering $p_{X}: \hat{X} \rightarrow X$ induced from the covering $p_{Z}: \hat{Z} \rightarrow Z$ by the inclusion $X \subset Z$ is well defined. The covering space $\hat{X}$, which is the stratified manifold (with singularities), is decomposed into the union of submanifolds

$$
X_{0}=\mathbb{R} \mathrm{P}^{\frac{n}{2}+64} \times \mathbb{R} \mathrm{P}^{63}, \quad \ldots, \quad X_{i}=\mathbb{R} \mathrm{P}^{\frac{n}{2}-64(i-1)} \times \mathbb{R} \mathrm{P}^{64(i+1)-1}, \quad \ldots, \quad X_{\frac{n+2}{64}}=\mathbb{R P}^{63} \times \mathbb{R} \mathrm{P}^{\frac{n}{2}+64} .
$$

Each manifold $\hat{X}_{i}$ of the family is the 2-sheeted covering space over the manifold $X_{i}$ over the first coordinate. Let us define $d_{1}(i)=\frac{n}{2}-64(i-1)$ and $d_{2}(i)=64(i+1)-1$. Then the formula for $X_{i}$ is $X_{i}=\mathbb{R} \mathrm{P}^{d_{1}(i)} \times \mathbb{R P}^{d_{2}(i)}$.

In the cohomologies of $X$, the classes $\rho_{X, 1} \in H^{1}(X ; \mathbb{Z} / 4)$ and $\kappa_{X, 2} \in H^{1}(X ; \mathbb{Z} / 2)$ are well defined. These classes are induced by the generators of the groups $H^{1}(Z ; / Z / 4)$ and $H^{1}(Z ; \mathbb{Z} / 2)$. Similarly, the cohomology classes $\kappa_{\hat{X}, i} \in H^{1}(\hat{X} ; \mathbb{Z} / 4), i=1,2$, are well defined. The cohomological class $\kappa_{\hat{X}, 1}$ is induced by the class $\rho_{X, 1} \in H^{1}(X ; \mathbb{Z} / 4)$ by means of the transfer homomorphism, and $\kappa_{\hat{X}, 2}=\left(p_{X}\right)^{*}\left(\kappa_{X, 2}\right)$.

For an arbitrary $j=0, \ldots, \frac{n+2}{128}$, define the space $J_{j}$; the mapping $\varphi_{j}: X_{j} \rightarrow J_{j}$ is well defined. We denote by $Y_{1}(k)$ the space $S^{63} / i * \cdots * S^{63} / i$ of the join of $k$ copies, $k=1, \ldots, \frac{n+2}{128}+1$, of the standard lens space $S^{63} / i$. Let us denote by $Y_{2}(k), k=1, \ldots, \frac{n+2}{128}+1, Y_{2}(k)=\mathbb{R P} \mathrm{P}^{63} * \cdots * \mathbb{R P}^{63}$, the joins of $k$ copies of the standard projective space $\mathbb{R P}^{63}$. We set $J_{j}=Y_{1}\left(\frac{n+2}{128}-j+1\right) \times Y_{2}(j+1)$ and $Q=Y_{1}\left(\frac{n+2}{128}+1\right) \times Y_{2}\left(\frac{n+2}{128}+1\right)$. For a given $j$, the natural inclusions $J_{j} \subset Q$ are well defined. Denote the union of the considered inclusions by $J$.

The mapping $\varphi_{j}: X_{j} \rightarrow J_{j}$ is well defined as the Cartesian product of the following two mappings. Over the first coordinate, the mapping is defined as the composition of the standard 2-sheeted covering $\mathbb{R P}^{d_{1}(j)} \rightarrow S^{\frac{n}{2}-64(j-1)} / i$ and the natural projection $S^{d_{1}(j)} / i \rightarrow Y_{1}\left(\frac{n+2}{128}-j+1\right)$. Over the second coordinate, the mapping is defined by the natural projection $\mathbb{R P}^{d_{2}(j)} \rightarrow Y_{2}(j+1)$.

The family of the mapping $\varphi_{j}$ determines the mapping $\varphi: \hat{X} \rightarrow J$, since the restrictions of the two mappings on the common subspace in the origin coincide.

For $n+2 \geq 2^{15}$, the space $J$ is embeddable into the Euclidean $n$-space by an embedding $i_{J}: J \subset \mathbb{R}^{n}$. Each space $Y_{1}(k), Y_{2}(k)$ in the family is embeddable into the Euclidean $\left(2^{8} k-1-k\right)$-space. Therefore, for an arbitrary $j$, the space $J_{j}$ is embeddable into the Euclidean space of dimension $2^{8}\left(\frac{n+2}{128}+2\right)-\frac{n+2}{128}-2$. In particular, if $n+2 \geq 2^{15}$, the space $J_{j}$ is embeddable into $\mathbb{R}^{n}$. The image of an arbitrary intersection of two embeddings in the family belongs to the standard coordinate subspace. Therefore, the required embedding $i_{J}$ is defined by the gluing of embeddings in the family.

Describe the mapping $\hat{h}: \hat{X} \rightarrow \mathbb{R}^{n}$. We denote by $\varepsilon$ the radius of a (stratified) regular neighborhood of the subpolyhedron $i_{J}(J) \subset \mathbb{R}^{n}$. Consider small positive $\varepsilon_{1}, \varepsilon_{1} \ll \varepsilon$ (this constant will be defined below in the proof of Lemma 2) and a generic $\varepsilon_{1}$-deformation of the mapping $i_{J} \circ \varphi: \hat{X} \rightarrow J \subset \mathbb{R}^{n}$. The result of the deformation is denoted by $\hat{h}: \hat{X} \rightarrow \mathbb{R}^{n}$.

Define the positive integer $k$ by the equation $n-4 k=62$. In the prescribed regular homotopy class of an $\mathbf{I}_{b}$-controlled immersion $f: N^{n-2 k} \uparrow \rightarrow \mathbb{R}^{n}$, we construct another $\mathbf{I}_{b}$-controlled immersion $g: N^{n-2 k} \rightarrow \mathbb{R}^{n}$ with the self-intersection-points manifold $L^{n-4 k}$ that admits a $(\mathbb{Z} / 2 \oplus \mathbb{Z} / 4)$-structure.

Let the immersion $f$ be controlled over the embedded subpolyhedron $\psi_{P}: P \subset \mathbb{R}^{n}$. Let $\psi_{Q}: Q \rightarrow \hat{X}$ be a generic mapping such that $\kappa_{Q, i}=\psi_{Q} \circ \kappa_{\hat{X}, i}, i=1,2$. By the previous definition, the manifolds $N_{\text {int }}^{n-2 k}$ and $N_{\text {ext }}^{n-2 k}$ with the common boundary $N_{Q}^{n-2 k-1}, N^{n-2 k}=N_{\text {int }}^{n-2 k} \cup_{N_{Q}^{n-2 k-1}} N_{\text {ext }}^{n-2 k}$ are well defined.

Let $\eta: N_{\text {ext }}^{n-2 k} \rightarrow K\left(\mathbf{I}_{b}, 1\right) \subset K\left(\mathbf{D}_{4}, 1\right)$ be the characteristic mapping of the framing $\Xi_{N}$ restricted to $N_{\mathrm{ext}}^{n-2 k} \subset N^{n-2 k}$. The restriction of this mapping to the boundary $\partial N_{\mathrm{ext}}^{n-2 k}=N_{Q}^{n-2 k-1}$ is given by the composition $\partial N_{Q}^{n-2 k-1} \rightarrow Q \rightarrow K\left(\mathbf{I}_{b}, 1\right) \subset K\left(\mathbf{D}_{4}, 1\right)$. The target space for the mapping $\eta$ is the 
subspace $K\left(\mathbf{I}_{b}, 1\right) \subset K\left(\mathbf{D}_{4}, 1\right)$. This mapping is determined by the cohomological classes $\kappa_{N_{\mathrm{ext}}^{n-2 k}, i} \in$ $H^{1}\left(N_{\mathrm{ext}}^{n-2 k}, Q ; \mathbb{Z} / 2\right), i=1,2$.

Define the mapping $\lambda: N_{\text {ext }}^{n-2 k} \rightarrow \hat{X}$ by the following conditions. This mapping transforms the cohomological classes $\kappa_{\hat{X}, i}$ into the classes $\kappa_{i} \in H^{1}\left(N_{\text {ext }}^{n-2 k} ; \mathbb{Z} / 2\right)$ and also the restriction $\left.\lambda\right|_{N_{Q}^{n-2 k-1}}$ coincides with the composition of the projection $N_{Q}^{n-2 k-1} \rightarrow Q$ and the mapping $\psi_{Q}: Q \rightarrow \hat{X}$. The boundary conditions for the mapping $\psi_{Q}$ are $\kappa_{Q, i}=\psi_{Q} \circ \kappa_{\hat{X}, i}, i=1,2$.

Denote the composition $\hat{h} \circ \lambda: N_{\text {ext }}^{n-2 k} \rightarrow \hat{X} \rightarrow \mathbb{R}^{n}$ by $g_{1}$ and the mapping $\hat{h} \circ \psi_{Q}: Q \rightarrow \hat{X} \rightarrow \mathbb{R}^{n}$ by $\varphi_{Q}$. One can assume that the mapping $\varphi_{Q}$ is an embedding. Moreover, without loss of generality, one can assume that this embedding is extended to a generic embedding $\varphi_{P}: P \subset \mathbb{R}^{n}$ such that the embedded polyhedron $\varphi_{P}: P \subset \mathbb{R}^{n}$ is not intersected with $g_{1}\left(N_{\text {ext }}^{n-2 k}\right)$ outside $\varphi_{Q}(Q)$.

Denote by $U_{\varphi}(P)$ a regular neighborhood of the subpolyhedron $\varphi_{P}(P) \subset \mathbb{R}^{n}$ (we can assume that the radius of this neighborhood is equal to $\varepsilon$ ). Since an isotopy in a regular neighborhood $U_{\varphi}(P)$ is well defined, in particular, this neighborhood is independent of the choice of the regular embedding of $P$, $U_{\varphi}(P)$ and $U(P)$ are diffeomorphic.

The immersion $g^{\prime}: N_{\text {int }}^{n-2 k} \subset \mathbb{R}^{n}$ with the image inside $U_{\varphi}(P)$ is well defined. The restriction of $g^{\prime}$ on the boundary $N_{Q}^{n-2 k-1}=\partial\left(N_{\text {int }}^{n-2 k}\right)$ is a regular embedding $N_{Q}^{n-2 k-1} \subset \partial U(P)$. This immersion $g^{\prime}$ is conjugated with the immersion $\left.f\right|_{N_{\text {int }}}$ by means of a diffeomorphism of $U_{\varphi}(P)$ and $U(P)$. We can assume, without loss of generality, that the restriction $\left.g_{1}\right|_{N_{\text {int }}^{n-2 k}}$ corresponds to $g^{\prime}$.

By Proposition 2, for an arbitrary $\varepsilon_{2}>0, \varepsilon_{2} \ll \varepsilon_{1} \ll \varepsilon$, there exists an immersion $g: N^{n-2 k} q \mathbb{R}^{n}$ in the regular homotopy class of $f$ such that $g$ coincides with $g^{\prime}$ (and with $g_{1}$ ) on $N_{\text {int }}^{n-2 k}$ and, moreover, $\operatorname{dist}\left(g ; g_{1}\right)<\varepsilon_{2}$.

Let us consider the self-intersection manifold $L^{n-4 k}$ of the immersion $g$. This manifold is a submanifold in $\mathbb{R}^{n}$. Let us start with the construction of the mappings $\kappa_{a}: L^{n-4 k} \rightarrow K(\mathbb{Z} / 2,1)$ and $\mu_{a}: L^{n-4 k} \rightarrow$ $K(\mathbb{Z} / 4,1)$. Then we verify conditions $(5)$ and $(6)$.

The manifold $L^{n-4 k}$ is naturally divided into two components. The first component $L_{\text {int }}^{n-4 k}$ is inside $U_{\varphi_{P}}(P)$. The last component (we denote this component again by $L^{n-4 k}$ ) consists of the last self-intersection points. This component is inside an $\varepsilon_{1}$-neighborhood of the submanifold with singularities $h(X)$. The mappings $\kappa_{a}$ and $\mu_{a}$ over $L_{\text {int }}^{n-4 k}$ are defined as the trivial mappings. Let us define the mappings $\kappa_{a}$ and $\mu_{a}$ on $L^{n-4 k}$.

Consider the mapping $\varphi: \hat{X} \rightarrow J$ and the singular set (polyhedron) $\Sigma$ of this mapping. This is the subpolyhedron

$$
\Sigma \subset\left\{\hat{X}^{(2)}=\hat{X} \times \hat{X} \backslash \Delta_{\hat{X}} / T^{\prime}\right\}
$$

where $T^{\prime}: \hat{X}^{(2)} \rightarrow \hat{X}^{(2)}$ is the involution of coordinates in the deleted product $\hat{X}^{(2)}$ of the space $\hat{X}$. The subpolyhedron $\Sigma$ (it is convenient to consider this polyhedron as a manifold with singularities) is naturally decomposed into the union of subpolyhedra $\Sigma(j), j=0, \ldots, \frac{n+2}{128}$. The subpolyhedron $\Sigma(j)$ is the singular set of the mapping

$$
\varphi(j): \mathbb{R} \mathrm{P}^{d_{1}(j)} \times \mathbb{R} \mathrm{P}^{d_{2}(j)} \rightarrow S^{d_{1}(j)} / i \times \mathbb{R} \mathrm{P}^{d_{2}(j)} \rightarrow J_{j}
$$

This subpolyhedron consists of the singular points of the mapping $\varphi$ in the inverse image $(\varphi)^{-1}\left(J_{j}\right)=$ $\mathbb{R P}^{d_{1}(j)} \times \mathbb{R P}^{d_{2}(j)}$ of the subspace $J_{j} \subset J$.

Consider the subspace $\Sigma^{\text {reg }} \subset \Sigma$, consisting of points on strata of length 0 (regular strata) and length 1 (singular strata of codimension 64 ) after the regular $\varepsilon_{2}$-neighborhoods $\left(\varepsilon_{2} \ll \varepsilon_{1}\right)$ of the diagonal $\Delta^{\text {diag }}$ and the antidiagonal $\Delta^{\text {antidiag }}$ of $\Sigma^{\text {reg }}$ will be cut out.

The manifold with singularities $\Sigma^{\text {reg }}$ admits a natural cementification (closure) in the neighborhood of $\Delta^{\text {diag }}$ and $\Delta^{\text {antidiag }}$, the result of the cementification being denoted by $K$.

The resolution mapping $R K \rightarrow K$ is defined by a similar construction (see [2, Lemma 7]). The cohomological classes $\rho_{R K, 1} \in H^{1}(R K ; \mathbb{Z} / 4)$ and $\kappa_{R K, 2} \in H^{1}(R K ; \mathbb{Z} / 2)$ are well defined. The cohomological 
classes $\kappa_{K, 1} \in H^{1}(R K ; \mathbb{Z} / 2)$ and $\kappa_{R K, 1} \in H^{1}(R K ; \mathbb{Z} / 2)$ are the images of the class $\kappa_{\Sigma, 1} \in H^{1}(\Sigma ; \mathbb{Z} / 2)$ with respect to the inclusion $K \subset \Sigma$ and the projection $R K \rightarrow \Sigma$. The class classifies the transposition of the two nonordered preimages of a point in the singular set.

Consider the restrictions of the classes $\kappa_{K, 1}, \kappa_{R K, 1}$, and $\kappa_{\Sigma, 1}$ on the neighborhood of the diagonal and the antidiagonal. The natural projection $\Delta^{\operatorname{diag}} \rightarrow \hat{X}$ is well defined. The restriction of the classes $\rho_{1}$ and $\kappa_{2}$ on the neighborhoods of the diagonal coincides with the restrictions of the classes $\rho_{\hat{X}, 1} \in H^{1}(\hat{X} ; \mathbb{Z} / 4)$ and $\kappa_{\hat{X}, 2} \in H^{1}(\hat{X} ; \mathbb{Z} / 2)$ (these classes are extended to the regular neighborhoods of the diagonal).

Recall that the mapping $\hat{h}: \hat{X} \rightarrow \mathbb{R}^{n}$ is defined as the result of an $\varepsilon_{1}$-small regular deformation of the mapping $\hat{X} \longrightarrow X \stackrel{h}{\longrightarrow} \mathbb{R}^{n}$. The singular set of the mapping $\hat{h}$ is denoted by $\Sigma_{\hat{h}}$. This is a 128-dimensional polyhedron, or the manifold with singularities in codimensions 32, 64, 96, and 128. Moreover, the inclusion $\Sigma_{\hat{h}} \subset \hat{X}^{(2)}$ is well defined. The image of this inclusion is in the regular $\varepsilon_{1}$-small neighborhood of the singular polyhedron $\Sigma \subset X^{(2)}$.

Denote by $\Sigma_{\hat{h}}^{\text {reg }}$ the part of the singular set after the cutting-out of the regular $\varepsilon_{1}$-neighborhood of the points in singular strata of length at least 2 (of codimension 64) and self-intersection points of all singular strata (these strata are also of codimension 64). The boundary $\partial \Sigma_{\hat{h}}$ is a submanifold with singularities in $\hat{X}$ and, therefore, we can also assume that the boundary $\partial \Sigma_{\hat{h}}^{\mathrm{reg}}$ is a regular submanifold with singularities in $\hat{X}$.

By general-position arguments, the intersection of the projection $\hat{X} \rightarrow X$ of double points of the image $\operatorname{Im}\left(\lambda\left(N_{\text {ext }}^{n-2 k}\right)\right)$ with the projection of the singular set $\Sigma_{\hat{h}}$ (this is a subpolyhedron of dimension 62) on $X$ is outside of the projection of the submanifold with singularities $\Sigma_{\hat{h}}^{\mathrm{reg}} \subset \Sigma_{\hat{h}}$ (this is the submanifold with singularities of codimension 64 ).

Denote by $L_{\text {cycl }}^{62} \subset L^{62}$ the submanifold (with boundary) given by the formula $L_{\text {cycl }}^{62}=L^{62} \cap U_{\Sigma^{\text {reg }}}$. The mappings $\kappa_{a}$ and $\rho_{a}$ are extendable from $U_{\Sigma^{\text {reg }}}$ to $L_{\mathrm{cycl}}^{62}$. Let us prove that these mappings are extendable to the mappings $\kappa_{a}: L^{62} \rightarrow K(\mathbb{Z} / 2,1)$ and $\rho_{a}: L^{62} \rightarrow K(\mathbb{Z} / 4,1)$.

The complement of this submanifold is denoted by $L_{\mathbf{I}_{3}}^{62}=L^{62} \backslash L_{\text {cycl }}^{62}$. The submanifold $L_{\mathbf{I}_{3}}^{62}$ is a submanifold in the regular $\varepsilon$-neighborhood of $h(X) \subset \mathbb{R}^{n}$. Obviously, the structure group of the $\left(\mathbb{Z} / 2 \prec \mathbf{D}_{4}\right)$-framing of the normal bundle of the manifold $L_{\mathbf{I}_{3}}^{62}$ is reduced to the subgroup $\mathbf{I}_{3} \subset \mathbb{Z} / 2 \imath \mathbf{D}_{4}$.

Consider the mapping of pairs

$$
\mu_{a} \times \kappa_{a}:\left(L_{\text {cycl }}^{62}, \partial L_{\text {cycl }}^{62}\right) \rightarrow(K(\mathbb{Z} / 4,1) \times K(\mathbb{Z} / 2,1), K(\mathbb{Z} / 2,1) \times K(\mathbb{Z} / 2,1)) .
$$

Consider the natural projection $\pi_{b}: \mathbf{I}_{3} \rightarrow \mathbf{I}_{b}$. The extension of the mapping $\mu_{a} \times \kappa_{a}$ to the required mapping $L^{62} \rightarrow K(\mathbb{Z} / 4,1) \times K(\mathbb{Z} / 2,1)$ is given by the composition

$$
L_{\mathbf{I}_{3}}^{62} \longrightarrow K\left(\mathbf{I}_{3}, 1\right) \stackrel{\pi_{b, *}}{\longrightarrow} K\left(\mathbf{I}_{b}, 1\right) \subset K(\mathbb{Z} / 4,1) \times K(\mathbb{Z} / 2,1),
$$

where $\kappa_{1} \in K\left(\mathbf{I}_{b} ; \mathbb{Z} / 2\right)$ determines the inclusion $K\left(\mathbf{I}_{b}, 1\right) \subset K(\mathbb{Z} / 2,1) \subset K(\mathbb{Z} / 4,1)$.

Let us state the results in the following lemma.

\section{Lemma 2.}

(1) Let $n \geq 2^{16}-2$ and $k, n-4 k=62$, be under the conditions of Theorem 1 . Then for arbitrarily small positive $\varepsilon_{1}$ and $\varepsilon_{2}$ (the diameters of the regular deformations in the construction of the mapping $\hat{h}: \hat{X} \rightarrow \mathbb{R}^{n}$ and of the immersion $\left.g: N^{n-2 k} \rightarrow \mathbb{R}^{n}\right)$, there exists the mapping

$$
m_{a}=\left(\kappa_{a} \times \mu_{a}\right): \Sigma_{h}^{\mathrm{reg}} \rightarrow K(\mathbb{Z} / 4,1) \times K(\mathbb{Z} / 2,1)
$$

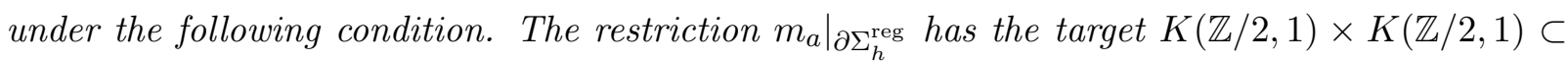
$K(\mathbb{Z} / 4,1) \times K(\mathbb{Z} / 2,1)$ and is determined by the cohomological classes $\kappa_{\hat{X}, 1}$ and $\kappa_{\hat{X}, 2}$.

(2) The mappings $\kappa_{a}$ and $\mu_{a}$ induce the mappings $\left(\mu_{a} \times \kappa_{a}\right): L^{62} \rightarrow K(\mathbb{Z} / 4,1) \times K(\mathbb{Z} / 2,1)$ on the self-intersection manifold of the immersion $g$. 
Let us prove that the mapping $\left(\mu_{a} \times \kappa_{a}\right)$ constructed in Lemma 2 determines a $(\mathbb{Z} / 2 \oplus \mathbb{Z} / 4)$-structure for the $\mathbf{D}_{4}$-framed immersion immersion $g$. We must prove Eq. (6).

Recall that the component $L_{\text {int }}^{62}$ of the self-intersection manifold of the immersion $g$ is a $\left(\mathbb{Z} / 2<\mathbf{D}_{4}\right)$-framed manifold with the trivial Kervaire invariant: the corresponding element in the group

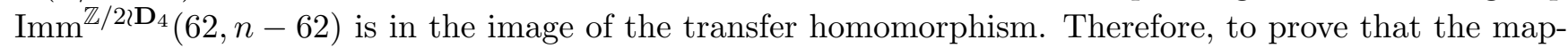
ping $m_{a}$ determines a $(\mathbb{Z} / 2 \oplus \mathbb{Z} / 4)$-structure, it suffices to prove the equation

$$
\left\langle m_{a}^{*}\left(\rho \tau^{15} t^{31}\right) ;\left[L^{62}\right]\right\rangle=\Theta
$$

or, equivalently, the equation

$$
\left\langle\left(\hat{\rho}_{a}^{31} \hat{\kappa}_{a}^{31}\right) ;\left[\hat{L}^{62}\right]\right\rangle=\Theta,
$$

where $\hat{L} \rightarrow L$ is the canonical cover over the self-intersection-points manifold and $\hat{L} \subset N_{\text {ext }}^{n-2 k}$ is the canonical inclusion.

By the Herbert theorem (see $[1,2]$ for the analogous construction), we can calculate the right-hand side of the equation by the formula

$$
\left\langle\eta^{*}\left(w_{2}\left(\mathbf{I}_{b}\right)\right)^{\frac{n-2 k}{2}} ;\left[N_{\mathrm{ext}}^{n-2 k} / \sim\right]\right\rangle .
$$

In this formula, $N_{\mathrm{ext}}^{n-2 k} / \sim$ denotes the quotient of the boundary $\partial N_{\mathrm{ext}}^{n-2 k}=N_{Q}^{n-2 k-1}$ that is contracted onto the polyhedron $Q$ with the loss of dimension.

Note that the mapping $\left.m_{a}\right|_{N_{Q}^{n-2 k-1}}$ is obtained by the composition of the shrink $p_{Q}: N^{n-2 k-1} \rightarrow Q$ with the mapping $Q \rightarrow K\left(\mathbf{I}_{b}, 1\right)$, the last mapping being determined by the cohomological classes $\kappa_{i, Q} \in H^{1}(Q ; \mathbb{Z} / 2), i=1,2$. Therefore, $m_{a *}\left(\left[N_{\mathrm{ext}}^{n-2 k} / \sim\right]\right) \in H_{n-2 k}\left(\mathbf{I}_{b} ; \mathbb{Z} / 2\right)$ is a permanent cycle and the integration over the cycle $\left[N_{\mathrm{ext}}^{n-2 k} / \sim\right]$ of the inverse image of the universal cohomological class in (8) is well defined.

It is convenient to consider the characteristic number $\Theta_{a}$ as the value of a homomorphism $H_{n-2 k}(X ; \mathbb{Z} / 2) \rightarrow \mathbb{Z} / 2$ on the cycle $\lambda_{*}\left[N_{\text {ext }}^{n-2 k} / \sim\right] \in H_{n-2 k}(X ; \mathbb{Z} / 2)$. This homomorphism is the result of the calculation of the characteristic class $w_{2}\left(\mathbf{I}_{b}\right) \in H^{2}\left(K\left(\mathbf{I}_{b}, 1\right) ; \mathbb{Z} / 2\right)$ on the prescribed cycle, i.e., on the image of the fundamental cycle $\left[N_{\mathrm{ext}}^{n-2 k} / \sim\right]$ with respect to the mapping $N_{\mathrm{ext}}^{n-2 k} / \sim \rightarrow \hat{X} \rightarrow K\left(\mathbf{I}_{b}, 1\right)$. The cycle $\lambda_{*}\left[N_{\mathrm{ext}}^{n-2 k} / \sim\right] \in H_{n-2 k}(X ; \mathbb{Z} / 2)$ is integer. Therefore, this cycle is given by the sum of fundamental classes of the product of two odd-dimensional projective spaces, the sum of the dimensions of these spaces being equal to $n-2 k$.

Consider an arbitrary submanifold $S^{k_{1}} / i \times \mathbb{R P}^{k_{2}} \subset X, k_{1}+k_{2}=\frac{n}{2}+31, k_{1}$ and $k_{2}$ are odd. Consider the cover $\mathbb{R} \mathrm{P}^{k_{1}} \times \mathbb{R} \mathrm{P}^{k_{2}} \rightarrow S^{k_{1}} / i \times \mathbb{R} \mathrm{P}^{k_{2}}$ and the composition $\mathbb{R} \mathrm{P}^{k_{1}} \times \mathbb{R} \mathrm{P}^{k_{2}} \subset \hat{X} \stackrel{\hat{h}}{\rightarrow} \mathbb{R}^{n}$ after an $\varepsilon_{1}$-small generic perturbation. Denote this mapping by $s_{k_{1}, k_{2}}$.

The self-intersection manifold of the generic mapping $s_{k_{1}, k_{2}}: \mathbb{R P}^{k_{1}} \times \mathbb{R} \mathrm{P}^{k_{2}} \rightarrow \mathbb{R}^{n}$ is a manifold with boundary denoted by $\Lambda_{k_{1}, k_{2}}^{62}$. The mapping

$$
\mu_{a} \times \kappa_{a}:\left(\Lambda_{k_{1}, k_{2}}^{62}, \partial N_{k_{1}, k_{2}}^{n-2 k}\right) \rightarrow(K(\mathbb{Z} / 4,1) \times K(\mathbb{Z} / 2,1), K(\mathbb{Z} / 2,1) \times K(\mathbb{Z} / 2,1))
$$

is well defined. The homological 61 -dimensional fundamental class $[\partial \Lambda]$ is integer; therefore, the image of this fundamental class

$$
\left(\mu_{a} \times \kappa_{a}\right)_{*}\left(\left[\partial \Lambda_{k_{1}, k_{2}}^{62}\right]\right) \in H_{61}(K(\mathbb{Z} / 4,1) \times K(\mathbb{Z} / 2,1) ; \mathbb{Z} / 2)
$$

is trivial by the dimensional reason. Therefore, the homological class

$$
\left(\mu_{a} \times \kappa_{a}\right)_{*}\left(\left[\Lambda_{k_{1}, k_{2}}^{62}, \partial \Lambda_{k_{1}, k_{2}}^{62}\right]\right) \in H_{62}(K(\mathbb{Z} / 4,1) \times K(\mathbb{Z} / 2,1), K(\mathbb{Z} / 2,1) \times K(\mathbb{Z} / 2,1) ; \mathbb{Z} / 2)
$$

is well defined. Let us consider the (permanent) homological class

$$
\left(\mu_{a} \times \kappa_{a}\right)_{*}^{!}\left(\left[\bar{\Lambda}_{k_{1}, k_{2}}^{62}\right]\right) \in H_{62}(K(\mathbb{Z} / 2,1) \times K(\mathbb{Z} / 2,1) ; \mathbb{Z} / 2)
$$

defined from the relative class above by the transfer homomorphism. 
To prove (7), it suffices to prove that the class (9) coincides with the characteristic class $p_{*, b} \circ \hat{\eta}_{*}([\hat{\Lambda}]) \in$ $H_{62}\left(K\left(\mathbf{I}_{b}, 1\right) ; \mathbb{Z} / 2\right)$ under the isomorphism of the target group $\mathbf{I}_{b}=\mathbb{Z} / 2 \oplus \mathbb{Z} / 2$. By this isomorphism, the prescribed generators in $H^{1}(\mathbb{Z} / 2 \oplus \mathbb{Z} / 2 ; \mathbb{Z} / 2)$ are identified with the cohomological classes $\tau_{1}, \tau_{2} \in$ $H^{1}\left(K\left(\mathbf{I}_{b}, 1\right) ; \mathbb{Z} / 2\right)$ (cf. [2, Lemma 8]). Theorem 2 is proved.

This work was partially supported by the London Royal Society (1998-2000), the Russian Foundation for Basic Research (project No. 08-01-00663), and INTAS (project No. 05-1000008-7805).

\section{REFERENCES}

1. P. M. Akhmet'ev, Geometric Approach to Stable Homotopy Groups of Spheres. The Hopf Invariant, arXiv:0710.5779[math.AT].

2. P. M. Akhmet'ev, Geometric Approach towards Stable Homotopy Groups of Spheres. Kervaire Invariant [in Russian], arXiv:0710.5853[math.GT].

3. P. M. Akhmet'ev and P. J. Eccles, "The relationship between framed bordism and skew-framed bordism," Bull. London Math. Soc., 39, No. 3, 473-481 (2007).

4. M. G. Barratt, J. D. S. Jones, and M. E. Mahowald, "The Kervaire invariant problem," Contemp. Math., 19, 9-22 (1983).

5. J. S. Carter, "Surgery on codimension one immersions in $\mathbb{R}^{n+1}$ : Removing $n$-tuple points," Trans. Amer. Math. Soc., 298, No. 1, 83-101 (1986).

6. J. S. Carter, "On generalizing Boy's surface: Constructing a generator of the third stable stem," Trans. Amer. Math. Soc., 298, No. 1, 103-122 (1986).

7. R. L. Cohen, J. D. S. Jones, and M. E. Mahowald, "The Kervaire invariant of immersions," Invent. Math., 79, 95-123 (1985).

8. P. J. Eccles, "Codimension one immersions and the Kervaire invariant one problem," Math. Proc. Cambridge Phil. Soc., 90, 483-493 (1981).

9. P. J. Eccles, "Representing framed bordism classes by manifolds embedded in low codimension," in: Geom. Appl. Homotopy Theory I, Proc. Conf., Evanston 1977, Lect. Notes Math., Vol. 657, Springer, Berlin (1978), pp. 150-155.

10. M. Gromov, Partial Differential Relations, Ergebnisse Math. ihrer Grenzgebiete 3 Folge, Springer, Berlin (1986).

11. A. Szucs, "Topology of $\Sigma^{1,1}$-singular maps," Math. Proc. Cambridge Philos. Soc., 121, No. 3, 465-477 (1997).

P. M. Akhmet'ev

Steklov Mathematical Institute, Russian Academy of Sciences, Moscow, Russia

E-mail: pmakhmet@mi.ras.ru 